\title{
Towards the quantization of the non-relativistic D2-brane in the pure spinor formalism
}

\author{
A. Herrera-Aguilar ${ }^{1,2, a} \mathbb{C}$, J. E. Paschalis ${ }^{3, b}$ \\ ${ }^{1}$ Instituto de Física, Benemérita Universidad Autónoma de Puebla, Apartado Postal J-48, 72570 Puebla, Puebla, Mexico \\ ${ }^{2}$ Instituto de Física y Matemáticas, Universidad Michoacana de San Nicolás de Hidalgo, Edificio C-3, Ciudad Universitaria, CP 58040 Morelia, \\ Michoacán, Mexico \\ ${ }^{3}$ Theoretical Physics Department, Aristotle University of Thessaloniki, 54124 Thessaloníki, Greece
}

Received: 18 March 2019 / Accepted: 26 May 2019 / Published online: 18 June 2019

(C) The Author(s) 2019

\begin{abstract}
An attempt is made to apply the pure spinor formalism to the non-relativistic IIA D2-brane. The fermionic constraints corresponding to the rescaled fermionic coordinates are given. Two commuting spinor fields are introduced, each one corresponding to a fermionic constraint. A BRST charge is constructed via the ansatz proposed by Berkovits in (JHEP 04:018, arXiv:hep-th/0001035, 2000, JHEP 09:046, arXiv:hep-th/0006003, 2000, JHEP 09:016, arXiv:hep-th/0105050, 2001, ICTP Lect. Notes Ser. 13:57, arXiv:hep-th/0209059, 2003). The nilpotency of the BRST charge leads to a set of constraints for the two spinor fields including pure spinor constraints. A novel non-trivial solution is given for one of the spinor fields which can be written as a sum of two pure spinors.
\end{abstract}

\section{Introduction}

The pure spinor formalism introduced by Berkovits [1-4] is a successful attempt to solve the longstanding problem of finding a manifestly supersymmetric and covariant superstring formalism. The basic ingredient is the BRST-like operator $Q=\int d z \lambda^{\alpha} d_{\alpha}$ where $d_{\alpha}$ is the fermionic constraint that appears in the conventional Green-Schwarz formalism and $\lambda_{\alpha}$ is a bosonic chiral spinor that plays the role of the associated "ghost". For $Q$ to be regarded as a BRST operator must be nilpotent and this leads to the relation $\lambda^{\alpha} \gamma_{\alpha \beta}^{m} \lambda^{\beta}=0$. This in 10 dimensions is the condition for $\lambda^{\alpha}$ to be characterized as a pure spinor.

An important property of $Q$ is that its cohomology correctly reproduces the spectrum of the superstring. The pure spinor formalism has been used as well for the covariant

\footnotetext{
a e-mail: aherrera@ifuap.buap.mx

be-mail: paschalis@physics.auth.gr
}

quantization of the superparticle [3] and also to study several aspects of string theory, for example the propagation of strings in curved backgrounds [5-8].

Another important application of the pure spinor formalism is the calculation of scattering amplitudes within the framework of superstring theory [9-13]. The manifest Lorentz covariance and spacetime supersymmetry make the calculation much easier than in other formalisms. Thus, pure spinors play a crucial role within string perturbation theory. However, within the context of D-branes, there are no nontrivial solutions reported in the literature. Moreover, so far it is not known whether this formalism can be consistently extended or generalized to be applied to non-relativistic systems with kappa symmetry, a fact that could allow us to draw some conclusions for the general case too, especially if there are problems in solving the pure spinor constraints in that case.

In this paper we try to extend the pure spinor formalism to the case of the non-relativistic IIA D2-brane. The non-relativistic limit of string theories $[14,15]$ give us a deeper understanding of string theories themselves. The nonrelativistic limit of Dp branes has been studied in $[16,17]$. It is important to note that in this limit the kappa symmetry is maintained and this allows us to treat non-relativistic Dp branes in the framework of the pure spinor formalism.

Here we present a novel non-trivial solution for the nonrelativistic D2-brane within the pure spinor formalism. This fact could lead to the quantization of branes with interesting and relevant results.

Our starting point is the action of a IIA D2-brane in a flat 10d background. The fields consist of the $10 \mathrm{~d}$ superspace coordinates $\left(x^{m}, \theta\right)$ and an Abelian gauge field $A_{\mu}[18-20]$ :

$S=-T \int d^{3} \sigma \sqrt{-\operatorname{det}\left(G_{\mu \nu}+\mathcal{F}_{\mu \nu}\right)}+\int \mathcal{L}_{3}^{W Z}$, 
where $T$ is the string tension and the Wess-Zumino action reads

$\mathcal{L}_{3}^{W Z}=T\left\{\frac{1}{2} d \bar{\theta} \not \AA^{2} \theta-\frac{1}{3} d \bar{\theta} \not \theta_{1}+\frac{1}{15} d \bar{\theta} \theta_{2}+d \bar{\theta} \Gamma_{11} \theta \mathcal{F}\right\} ;$

$G_{\mu \nu}=\eta_{m n} \Pi_{\mu}^{m} \Pi_{v}^{n}$ and here $\Pi_{\mu}^{m}=\frac{\partial X^{m}}{\partial \sigma^{\mu}}-\bar{\theta} \Gamma^{m} \frac{\partial \theta}{\partial \sigma^{\mu}}$.

$\begin{aligned} \mathcal{F}_{\mu \nu}= & \frac{\partial A_{\nu}}{\partial \sigma^{\mu}}-\frac{\partial A_{\mu}}{\partial \sigma^{\nu}}+\left(\bar{\theta} \Gamma_{11} \Gamma_{m} \partial_{\nu} \theta\right)\left(\Pi_{\mu}^{m}+\frac{1}{2} \bar{\theta} \Gamma^{m} \partial_{\mu} \theta\right) \\ & -\left(\bar{\theta} \Gamma_{11} \Gamma_{m} \partial_{\mu} \theta\right)\left(\Pi_{v}^{m}+\frac{1}{2} \bar{\theta} \Gamma^{m} \partial_{\nu} \theta\right)\end{aligned}$

$\theta_{1}=\bigvee \theta, \quad \theta_{2}=\tilde{\nabla} \nabla \theta$

with

$\bigvee=\left\{\left(\bar{\theta} \Gamma^{m} d \theta\right)+\Gamma_{11}\left(\bar{\theta} \Gamma_{11} \Gamma^{m} d \theta\right)\right\} \Gamma_{m}$

$\widetilde{V}=\left\{\left(\bar{\theta} \Gamma^{m} d \theta\right)-\Gamma_{11}\left(\bar{\theta} \Gamma_{11} \Gamma^{m} d \theta\right)\right\} \Gamma_{m}$

$\not \Lambda=\left(d X^{m}+\bar{\theta} \Gamma^{m} d \theta\right) \Gamma_{m}$

$\mathcal{F}=\frac{1}{2} \mathcal{F}_{\mu \nu} d \sigma^{\mu} d \sigma^{\nu}$

and $m, n=0, \ldots, 9 / \mu, v=0,1,2$.

The action (1.1) has a global supersymmetry and also a local supersymmetry (kappa symmetry).

The action for the non-relativistic D2-brane is obtained from (1.1) by doing the following rescaling [16,17]:

$$
\begin{aligned}
& X^{\mu}=\omega x^{\mu} \\
& X^{a}=x^{a} \\
& T=\frac{1}{\omega} T_{N R} \\
& A_{i}=\omega w_{i}
\end{aligned}
$$

where the subindex ${ }_{N R}$ stands for non-relativistic and $i, \mu=$ $0,1,2 / a=3, \ldots, 9$.

$\theta=\sqrt{\omega} \theta_{-}+\frac{1}{\sqrt{\omega}} \theta_{+}$

where $\theta_{+}, \theta_{-}$are eigenstates of

$$
\begin{aligned}
& \Gamma_{\star} \equiv \Gamma_{0} \Gamma_{1} \Gamma_{2} \\
& \Gamma_{\star} \theta_{ \pm}= \pm \theta_{ \pm}
\end{aligned}
$$

The action of the non-relativistic D2-brane is obtained by expanding (1.1) in powers of $\omega$ and keeping the finite part as $\omega \rightarrow \infty[17]$

$$
S_{N R}=\int d^{3} \sigma \mathcal{L}_{N R}^{D B I}+\int \mathcal{L}_{N R}^{W Z}
$$

where

$$
\begin{aligned}
\mathcal{L}_{N R}^{D B I}= & T_{N R}\left(\epsilon_{i j k} R_{i}^{0} R_{j}^{1} R_{k}^{2}\right)\left[-\left(\bar{\theta}_{+} \widehat{\gamma^{0}} \frac{\partial \theta_{+}}{\partial \sigma^{0}}+\bar{\theta}_{+} \widehat{\gamma^{1}} \frac{\partial \theta_{+}}{\partial \sigma^{1}}\right.\right. \\
& \left.+\bar{\theta}_{+} \widehat{\gamma^{2}} \frac{\partial \theta_{+}}{\partial \sigma 2}\right) \\
& \left.+\frac{1}{2} \widehat{g}^{i l}\left(\eta_{a a^{\prime}} u_{i}^{a} u_{l}^{a}\right)+\frac{1}{4} \widetilde{\mathcal{F}}_{i l}^{(1)} \widetilde{\mathcal{F}}_{j k}^{(1)} \widehat{g}^{i j} \widehat{g}^{l k}\right]
\end{aligned}
$$

$\mathcal{L}_{N R}^{W Z}=T_{N R}\left[\frac{1}{2}\left(\bar{\theta}_{+} \Gamma_{\mu \nu} d \theta_{+}\right)\left(\left(d x^{\mu}+\bar{\theta}_{-} \Gamma^{\mu} d \theta_{-}\right)\right.\right.$

$\left.\left(d x^{\nu}+\bar{\theta}_{-} \Gamma^{\nu} d \theta_{-}\right)-\left(\bar{\theta}_{-} \Gamma^{\mu} d \theta_{-}\right)\left(d x^{\nu}+\frac{2}{3} \bar{\theta}_{-} \Gamma^{\nu} d \theta_{-}\right)\right)$

$+\frac{1}{2}\left(\bar{\theta}_{-} \Gamma_{\mu \nu} d \theta_{-}\right)\left(\bar{\theta}_{+} \Gamma^{\mu} d \theta_{+}\right)\left(d x^{\nu}+\frac{2}{3} \bar{\theta}_{-} \Gamma^{\nu} d \theta_{-}\right)$

$+\frac{1}{2}\left(\bar{\theta}_{-} \Gamma_{a b} d \theta_{-}\right)\left(\left(d x^{a}+\bar{\theta}_{+} \Gamma^{a} d \theta_{-}+\bar{\theta}_{-} \Gamma^{a} d \theta_{+}\right)\right.$

$\times\left(d x^{b}+\bar{\theta}_{+} \Gamma^{b} d \theta_{-}+\bar{\theta}_{-} \Gamma^{b} d \theta_{+}\right)$

$-\left(\bar{\theta}_{+} \Gamma^{a} d \theta_{-}++\bar{\theta}_{-} \Gamma^{a} d \theta_{+}\right)$

$\left.\times\left(d x^{b}+\frac{2}{3}\left(\bar{\theta}_{-} \Gamma^{b} d \theta_{+}+\bar{\theta}_{+} \Gamma^{b} d \theta_{-}\right)\right)\right)$

$+\left(\bar{\theta}_{+} \Gamma_{\mu a} d \theta_{-}+\bar{\theta}_{-} \Gamma_{\mu a} d \theta_{+}\right)\left(\left(d x^{\mu}+\bar{\theta}_{-} \Gamma^{\mu} d \theta_{-}\right)\right.$

$\left.\times\left(d x^{a}+\frac{1}{2}\left(\bar{\theta}_{+} \Gamma^{a} d \theta_{-}+\bar{\theta}_{-} \Gamma^{a} d \theta_{+}\right)\right)\right)$

$-\frac{1}{2}\left(\bar{\theta}_{-} \Gamma^{\mu} d \theta_{-}\right)\left(d x^{a}+\frac{1}{3}\left(\bar{\theta}_{-} \Gamma^{a} d \theta_{+}+\bar{\theta}_{+} \Gamma^{a} d \theta_{-}\right)\right)$

$+\frac{1}{2}\left(\bar{\theta}_{+} \Gamma_{\mu} \Gamma_{11} d \theta_{-}+\bar{\theta}_{-} \Gamma_{\mu} \Gamma_{11} d \theta_{+}\right)$

$\times\left(d x^{\mu}+\frac{2}{3} \bar{\theta}_{-} \Gamma^{\mu} d \theta_{-}\right)\left(\bar{\theta}_{+} \Gamma_{11} d \theta_{-}+\bar{\theta}_{-} \Gamma_{11} d \theta_{+}\right)$

$+\frac{1}{2}\left(\bar{\theta}_{-} \Gamma_{b} \Gamma_{11} d \theta_{-}\right)\left(d x^{b}+\frac{2}{3}\left(\bar{\theta}_{-} \Gamma^{b} d \theta_{+}\right.\right.$

$\left.\left.+\bar{\theta}_{+} \Gamma^{b} d \theta_{-}\right)\right)\left(\bar{\theta}_{+} \Gamma_{11} d \theta_{-}+\bar{\theta}_{-} \Gamma_{11} d \theta_{+}\right)$

$+\left(d \bar{\theta}_{-} \Gamma_{11} \theta_{+}+d \bar{\theta}_{+} \Gamma_{11} \theta_{-}\right)\left(f-\left(\bar{\theta}_{-} \Gamma_{\mu} \Gamma_{11} d \theta_{+}\right.\right.$

$\left.+\bar{\theta}_{+} \Gamma_{\mu} \Gamma_{11} d \theta_{-}\right)\left(d x^{\mu}+\frac{1}{2} \bar{\theta}_{-} \Gamma^{\mu} d \theta_{-}\right)$

$\left.\left.-\left(\bar{\theta}_{-} \Gamma_{a} \Gamma_{11} d \theta_{-}\right)\left(d x^{a}+\frac{1}{2}\left(\bar{\theta}_{-} \Gamma^{a} d \theta_{+}+\bar{\theta}_{+} \Gamma^{a} d \theta_{-}\right)\right)\right)\right]$

where

$R_{i}^{\mu}=\partial_{i} x^{\mu}-\bar{\theta}_{-} \Gamma^{\mu} \partial_{i} \theta_{-}$

$u_{i}^{a}=\partial_{i} x^{a}-\bar{\theta}_{-} \Gamma^{a} \partial_{i} \theta_{+}-\bar{\theta}_{+} \Gamma^{a} \partial_{i} \theta_{-}$

$i, \mu=0,1,2 / a=3, \ldots, 9$; also

$$
\begin{aligned}
& \widehat{\gamma^{0}}=\frac{1}{\left(\epsilon_{i j k} R_{i}^{0} R_{j}^{1} R_{k}^{2}\right)}\left(\epsilon_{i j k} \Gamma^{i} R_{1}^{j} R_{2}^{k}\right) \\
& \widehat{\gamma^{1}}=-\frac{1}{\left(\epsilon_{i j k} R_{i}^{0} R_{j}^{1} R_{k}^{2}\right)}\left(\epsilon_{i j k} \Gamma^{i} R_{0}^{j} R_{2}^{k}\right)
\end{aligned}
$$




$$
\begin{aligned}
\widehat{\gamma^{2}} & =\frac{1}{\left(\epsilon_{i j k} R_{i}^{0} R_{j}^{1} R_{k}^{2}\right)}\left(\epsilon_{i j k} \Gamma^{i} R_{0}^{j} R_{1}^{k}\right) \\
\widehat{g}^{j k} & =\eta_{\mu \nu} R_{j}^{\mu} R_{k}^{\nu}
\end{aligned}
$$

where we have introduced the following quantities $\epsilon_{012}=1$, $\eta_{\mu \nu}=\operatorname{diag}(-1,1,1), \eta_{a a^{\prime}}=\operatorname{diag}(1, \ldots, 1), \mu, v=0,1,2$; $a, a^{\prime}=3, \ldots, 9$;

$$
\begin{aligned}
\widetilde{\mathcal{F}}_{i j}^{(1)}= & f_{i j}+\left[\left(\bar{\theta}_{-} \Gamma_{11} \Gamma_{\mu} \partial_{j} \theta_{+}+\bar{\theta}_{+} \Gamma_{11} \Gamma_{\mu} \partial_{j} \theta_{-}\right)\right. \\
& \left.\times\left(R_{i}^{\mu}+\frac{1}{2} \bar{\theta}_{-} \Gamma^{\mu} \partial_{i} \theta_{-}\right)-(i \leftrightarrow j)\right] \\
& +\left[( \overline { \theta } _ { - } \Gamma _ { 1 1 } \Gamma _ { a } \partial _ { j } \theta _ { - } ) \left(u_{i}^{a}+\frac{1}{2}\left(\bar{\theta}_{-} \Gamma^{a} \partial_{i} \theta_{+}\right.\right.\right. \\
& \left.\left.\left.+\bar{\theta}_{+} \Gamma^{a} \partial_{i} \theta_{-}\right)\right)-(i \leftrightarrow j)\right],
\end{aligned}
$$

where $f_{i j}=\partial_{i} w_{j}-\partial_{j} w_{i}, \quad f=\frac{1}{2} f_{i j} d \sigma^{i} d \sigma^{j}$, and $\mu, v, i, j=0,1,2 / a=3, \ldots, 9$.

We denote by $\left[\mathcal{L}_{N R}^{W Z}\right]_{3}$ the 3 -form coefficient of $\mathcal{L}_{N R}^{W Z}$ given in (1.18). The Lagrangian density of the non-relativistic D2brane is given by:

$$
\mathcal{L}_{N R}=\mathcal{L}_{N R}^{D B I}+\left[\mathcal{L}_{N R}^{W Z}\right]_{3}
$$

The action (1.16) is invariant under the non-relativistic counterpart of the global and local supersymmetric transformations that leave the relativistic D2-brane action (1.1) invariant [16].

The conjugate momenta of the variables $x^{\mu}, x^{a}, \theta_{+}, \theta_{-}$, $w_{i}$ are given by:

$$
\begin{aligned}
p_{\mu}= & \frac{\partial \mathcal{L}_{N R}}{\partial \dot{x}^{\mu}} \\
= & \frac{\partial \mathcal{L}_{N R}}{\partial R_{0}^{\mu}}+\frac{\partial \mathcal{L}_{N R}}{\partial \widetilde{\mathcal{F}}_{0 i}^{(1)}}\left(\bar{\theta}_{-} \Gamma_{11} \Gamma_{\mu} \partial_{i} \theta_{+}+\bar{\theta}_{+} \Gamma_{11} \Gamma_{\mu} \partial_{i} \theta_{-}\right) \\
\mu= & 0,1,2 / i=1,2 ; \\
p_{a}= & \frac{\partial \mathcal{L}_{N R}}{\partial \dot{x}^{a}} \\
= & \frac{\partial \mathcal{L}_{N R}}{\partial \dot{u}_{0}^{a}}+\frac{\partial \mathcal{L}_{N R}}{\partial \widetilde{\mathcal{F}}_{0 i}^{(1)}}\left(\bar{\theta}_{-} \Gamma_{11} \Gamma_{a} \partial_{i} \theta_{-}\right) \\
a=3, & \ldots, 9 ; \\
J_{+l}= & \frac{\partial^{r} \mathcal{L}_{N R}}{\partial \dot{\theta}_{+l}}-\frac{\partial \mathcal{L}_{N R}}{\partial u_{0}^{a}}\left(\bar{\theta}_{-} \Gamma^{a}\right)_{l} \\
& -\frac{\partial \mathcal{L}_{N R}}{\partial \widetilde{\mathcal{F}}_{0 i}^{(1)}}\left[\left(R_{i}^{\mu}+\frac{1}{2} \bar{\theta}_{-} \Gamma^{\mu} \partial_{i} \theta_{-}\right)\left(\bar{\theta}_{-} \Gamma_{11} \Gamma_{\mu}\right)_{l}\right. \\
& \left.+\frac{1}{2}\left(\bar{\theta}_{-} \Gamma_{11} \Gamma_{a} \partial_{i} \theta_{-}\right)\left(\bar{\theta}_{-} \Gamma^{a}\right)_{l}\right]
\end{aligned}
$$

and

$$
\begin{aligned}
J_{-l}= & \frac{\partial^{r} \mathcal{L}_{N R}}{\partial \dot{\theta}_{-l}}=\frac{\partial^{r}\left[\mathcal{L}_{N R}^{W Z}\right]_{3}}{\partial \dot{\theta}_{-l}}-\frac{\partial \mathcal{L}_{N R}}{\partial R_{0}^{\mu}}\left(\bar{\theta}_{-} \Gamma^{\mu}\right)_{l} \\
& -\frac{\partial \mathcal{L}_{N R}}{\partial u_{0}^{a}}\left(\bar{\theta}_{+} \Gamma^{a}\right)_{l} \\
& -\frac{\mathcal{L}_{N R}}{\partial \widetilde{\mathcal{F}}_{0 i}^{(1)}}\left[\frac{1}{2}\left(\bar{\theta}_{-} \Gamma_{11} \Gamma_{\mu} \partial_{i} \theta_{+}+\bar{\theta}_{+} \Gamma_{11} \Gamma_{\mu} \partial_{i} \theta_{-}\right)\left(\bar{\theta}_{-} \Gamma^{\mu}\right)_{l}\right. \\
& +\frac{1}{2}\left(\bar{\theta}_{-} \Gamma_{11} \Gamma_{a} \partial_{i} \theta_{-}\right)\left(\bar{\theta}_{+} \Gamma^{a}\right)_{l} \\
& +\left(R_{i}^{\mu}+\frac{1}{2} \bar{\theta}_{-} \Gamma^{\mu} \partial_{i} \theta_{-}\right)\left(\bar{\theta}_{+} \Gamma_{11} \Gamma_{\mu}\right)_{l} \\
& \left.+\left(u_{i}^{a}+\frac{1}{2}\left(\bar{\theta}_{-} \Gamma^{a} \partial_{i} \theta_{+}+\bar{\theta}_{+} \Gamma^{a} \partial_{i} \theta_{-}\right)\right)\left(\bar{\theta}_{-} \Gamma_{11} \Gamma_{a}\right)_{l}\right],
\end{aligned}
$$

where $l=1, \ldots, 32$; and

$E^{i}=\frac{\partial \mathcal{L}_{N R}}{\partial \dot{w}_{i}}=\frac{\partial \mathcal{L}_{N R}}{\partial \widetilde{\mathcal{F}}_{0 i}^{(1)}}$

The fermionic constraints are given by

$$
\begin{aligned}
F_{+l}= & J_{+l}+p_{a}\left(\bar{\theta}_{-} \Gamma^{a}\right)_{l} \\
& +E^{i}\left[\left(R_{i}^{\mu}+\frac{1}{2} \bar{\theta}_{-} \Gamma^{\mu} \partial \theta_{-}\right)\left(\bar{\theta}_{-} \Gamma_{11} \Gamma_{\mu}\right)_{l}\right. \\
& \left.-\frac{1}{2}\left(\bar{\theta}_{-} \Gamma_{11} \Gamma_{a} \partial_{i} \theta_{-}\right)\left(\bar{\theta}_{-} \Gamma^{a}\right)_{l}\right] \\
& +T_{N R} \epsilon_{i j k}\left(\bar{\theta}_{+} \Gamma^{i}\right)_{l} R_{1}^{j} R_{2}^{k}-\frac{\partial^{r}\left[\mathcal{L}_{N R}^{W Z}\right]_{3}}{\partial \dot{\theta}_{+l}}
\end{aligned}
$$

where the last derivative does not include differentiation with respect to $u_{0}^{a}, \widetilde{\mathcal{F}}_{0 i}^{(1)}$, and

$$
\begin{aligned}
F_{-l}= & J_{-l}+p_{a}\left(\bar{\theta}_{+} \Gamma^{a}\right)_{l}+p_{\mu}\left(\bar{\theta}_{-} \Gamma^{\mu}\right)_{l} \\
& +E^{i}\left[\left(R_{i}^{\mu}+\frac{1}{2}\left(\bar{\theta}_{-} \Gamma^{\mu} \partial_{i} \theta_{-}\right)\left(\bar{\theta}_{+} \Gamma_{11} \Gamma_{\mu}\right)_{l}\right.\right. \\
& +\left(u_{i}^{a}+\frac{1}{2}\left(\bar{\theta}_{-} \Gamma^{a} \partial_{i} \theta_{+}+\bar{\theta}_{+} \Gamma^{a} \partial_{i} \theta_{-}\right)\left(\bar{\theta}_{-} \Gamma_{11} \Gamma_{a}\right)_{l}\right. \\
& -\frac{1}{2}\left(\bar{\theta}_{-} \Gamma_{11} \Gamma_{\mu} \partial_{i} \theta_{+}+\bar{\theta}_{+} \Gamma_{11} \Gamma_{\mu} \partial_{i} \theta_{-}\right)\left(\bar{\theta}_{-} \Gamma^{\mu}\right)_{l} \\
& \left.-\frac{1}{2}\left(\bar{\theta}_{-} \Gamma_{11} \Gamma_{a} \partial_{i} \theta_{-}\right)\left(\bar{\theta}_{+} \Gamma^{a}\right)_{l}\right]-\frac{\partial^{r}\left[\mathcal{L}_{N R}^{W Z}\right]_{3}}{\partial \dot{\theta}_{-l}},
\end{aligned}
$$

where the last derivative does not include differentiation with respect to $u_{0}^{a}, R_{0}^{\mu}, \widetilde{\mathcal{F}}_{0 i}^{(1)}$.

We introduce now two commuting spinor fields $\lambda_{+}, \lambda_{-}$ corresponding to the fermionic constraints $F_{+}, F_{-}$and we write down a BRST charge according to the ansatz proposed in $[1-4]$ : 


$$
\begin{aligned}
Q= & \int d^{2} \sigma\left[\lambda_{+}^{l} J_{+l}+\lambda_{-}^{l} J_{-l}+p_{a}\left(\bar{\theta}_{-} \Gamma^{a} \lambda_{+}+\bar{\theta}_{+} \Gamma^{a} \lambda_{-}\right)\right. \\
& +p_{\mu}\left(\bar{\theta}_{-} \Gamma^{\mu} \lambda_{-}\right)+E^{i}\left[\left(R_{i}^{\mu}+\frac{1}{2} \bar{\theta}_{-} \Gamma^{\mu} \partial_{i} \theta_{-}\right)\right. \\
& \times\left(\bar{\theta}_{+} \Gamma_{11} \Gamma_{\mu} \lambda_{-}+\bar{\theta}_{-} \Gamma_{11} \Gamma_{\mu} \lambda_{+}\right)+\left(u_{i}^{a}+\frac{1}{2}\left(\bar{\theta}_{-} \Gamma^{a} \partial_{i} \theta_{+}\right.\right. \\
& \left.\left.+\bar{\theta}_{+} \Gamma^{a} \partial_{i} \theta_{-}\right)\right)\left(\bar{\theta}_{-} \Gamma_{11} \Gamma_{a} \lambda_{-}\right) \\
& -\frac{1}{2}\left(\bar{\theta}_{-} \Gamma_{11} \Gamma_{\mu} \partial_{i} \theta_{+}+\bar{\theta}_{+} \Gamma_{11} \Gamma_{\mu} \partial_{i} \theta_{-}\right)\left(\bar{\theta}_{-} \Gamma^{\mu} \lambda_{-}\right) \\
& \left.-\frac{1}{2}\left(\bar{\theta}_{-} \Gamma_{11} \Gamma_{a} \partial_{i} \theta_{-}\right)\left(\bar{\theta}_{+} \Gamma^{a} \lambda_{-}+\bar{\theta}_{-} \Gamma^{a} \lambda_{+}\right)\right] \\
& +T_{N R} \epsilon_{i j k}\left(\bar{\theta}_{+} \Gamma^{i} \lambda_{+}\right) R_{1}^{j} R_{2}^{k}-\left(\lambda_{+}^{T} \frac{\partial^{r}\left[\mathcal{L}_{N R}^{W Z}\right]_{3}}{\partial \dot{\theta}_{+}}\right) \\
& \left.-\left(\lambda_{-}^{T} \frac{\partial^{r}\left[\mathcal{L}_{N R}^{W Z}\right]_{3}}{\partial \dot{\theta}_{-}}\right)\right]
\end{aligned}
$$

with $i, j, k, \mu=0,1,2 / a=3, \ldots, 9$.

A set of BRST transformations is the following:

$$
\begin{aligned}
s \theta_{+}^{l}= & \lambda_{+}^{l}, \\
s \theta_{-}^{l}= & \lambda_{-}^{l}, \\
s x^{\mu}= & \bar{\theta}_{-} \Gamma^{\mu} \lambda_{-}, \\
s x^{a}= & \bar{\theta}_{-} \Gamma^{a} \lambda_{+}+\bar{\theta}_{+} \Gamma^{a} \lambda_{-}, \\
s R_{v}^{\mu}= & 2\left(\partial_{v} \bar{\theta}_{-} \Gamma^{\mu} \lambda_{-}\right), \\
s u_{\mu}^{a}= & 2\left(\partial_{\mu} \bar{\theta}_{-} \Gamma^{a} \lambda_{+}+\partial_{\mu} \bar{\theta}_{+} \Gamma^{a} \lambda_{-}\right), \\
s \widetilde{\mathcal{F}}_{i j}^{(1)}= & 2 R_{j}^{\mu}\left(\bar{\lambda}_{-} \Gamma_{11} \Gamma_{\mu} \partial_{i} \theta_{+}+\bar{\lambda}_{+}+\Gamma_{11} \Gamma_{\mu} \partial_{i} \theta_{-}\right) \\
& +2 u_{j}^{a}\left(\partial_{i} \bar{\theta}_{-} \Gamma_{11} \Gamma^{a} \lambda_{-}\right)-(i \leftrightarrow j)
\end{aligned}
$$

where again $\mu, v=0,1,2 / i, j=1,2 / a=3, \ldots, 9 / l=$ $1, \ldots 32$; from which we obtain

$$
\begin{aligned}
s^{2} \theta_{+}^{l} & =s^{2} \theta_{-}^{l}=0, \\
s^{2} x^{\mu} & =\bar{\lambda}_{-} \Gamma^{\mu} \lambda_{-}, \\
s^{2} x^{a} & =2\left(\bar{\lambda}_{-} \Gamma^{a} \lambda_{+}\right), \\
s^{2} R_{i}^{\mu} & =\frac{\partial}{\partial \sigma^{i}}\left(\bar{\lambda}_{-} \Gamma^{\mu} \lambda_{-}\right), \\
s^{2} u_{i}^{a} & =2 \frac{\partial}{\partial \sigma^{i}}\left(\bar{\lambda}_{+} \Gamma^{a} \lambda_{-}\right)
\end{aligned}
$$

and

$$
\begin{aligned}
s^{2} \widetilde{\mathcal{F}}_{i j}^{(1)}= & 2 R_{j}^{\mu} \frac{\partial}{\partial \sigma^{i}}\left(\bar{\lambda}_{+} \Gamma_{11} \Gamma_{\mu} \lambda_{-}\right)+u_{j}^{a} \frac{\partial}{\partial \sigma^{i}}\left(\bar{\lambda}_{-} \Gamma_{11} \Gamma_{a} \lambda_{-}\right) \\
& +2\left(\partial_{j} \bar{\theta}_{-} \Gamma_{11} \Gamma^{\mu} \partial_{i} \theta_{-}\right)\left(\bar{\lambda}_{-} \Gamma_{\mu} \lambda_{-}\right) \\
& +2\left(\partial_{j} \bar{\theta}_{-} \Gamma^{a} \partial_{i} \theta_{+}\right)\left(\bar{\lambda}_{-} \Gamma_{11} \Gamma_{a} \lambda_{-}\right) \\
& +2\left(\partial_{j} \bar{\theta}_{-} \Gamma_{11} \Gamma_{a} \partial_{i} \theta_{-}\right)\left(\bar{\lambda}_{-} \Gamma^{a} \lambda_{+}\right) \\
& +2\left(\partial_{j} \theta_{-} \Gamma^{\mu} \partial_{i} \theta_{-}\right)\left(\bar{\lambda}_{-} \Gamma_{11} \Gamma_{\mu} \lambda_{+}\right)
\end{aligned}
$$

where we used the ansatz

$$
\begin{aligned}
& \lambda_{-}=P_{-} \lambda, \\
& P_{-}=\frac{1}{2}\left(1-\Gamma_{0} \Gamma_{1} \Gamma_{2}\right)
\end{aligned}
$$

The full set of constraints required for the nilpotency of the BRST charge is obtained by studying the equal time Poisson bracket $\left\{Q(\sigma), Q\left(\sigma^{\prime}\right)\right\}$ where $Q(\sigma)$ is given in (1.34). A basis for expanding a $32 \times 32$ matrix $\mathrm{M}$ is given in Appendix A.

Thus, the expression for the equal time Poisson bracket $\left\{Q(\sigma), Q\left(\sigma^{\prime}\right)\right\}$ where $Q(\sigma)$ is the full BRST charge defined in (1.34) is given by:

$$
\begin{aligned}
\left\{Q(\sigma), Q\left(\sigma^{\prime}\right)\right\} \\
=\int d^{2} \sigma\left(\partial_{i} E^{i}\right)\left[\left(\bar{\theta}_{-} \Gamma^{a} \lambda_{+}+\bar{\theta}_{+} \Gamma^{a} \lambda_{-}\right)\left(\bar{\theta}_{-} \Gamma_{11} \Gamma_{a} \lambda_{-}\right)\right. \\
\left.\quad+\left(\bar{\theta}_{-} \Gamma^{\mu} \lambda_{-}\right)\left(\bar{\theta}_{+} \Gamma_{11} \Gamma_{\mu} \lambda_{-}+\bar{\theta}_{-} \Gamma_{11} \Gamma_{\mu} \lambda_{+}\right)\right] \\
\quad+2 T_{N R} \epsilon_{\mu \nu \rho} \int d^{2} \sigma R_{1}^{v} R_{2}^{\rho}\left(\bar{\lambda}_{+} \Gamma^{\mu} \lambda_{+}\right) \\
\quad+T_{N R} \epsilon_{0 j k} \int d \sigma^{2} R_{j}^{\mu} R_{k}^{v}\left(\bar{\lambda}_{+} \Gamma_{\mu \nu} \lambda_{+}\right) \\
\quad+\int d^{2} \sigma\left[F_{\mu}\left(\bar{\lambda}_{-} \Gamma^{\mu} \lambda_{-}\right)\right. \\
\quad+F_{a}\left(\bar{\lambda}_{-} \Gamma^{a} \Gamma_{11} \lambda_{-}\right)+F_{a b}\left(\bar{\lambda}_{-} \Gamma^{a b} \lambda_{-}\right) \\
\left.\quad+F^{v a b d f}\left(\bar{\lambda}_{-} \Gamma_{v a b d f} \lambda_{-}\right)+\bar{F}_{(} \bar{\lambda}_{-} \Gamma_{11} \lambda_{+}\right)+\bar{F}_{a}\left(\bar{\lambda}_{-} \Gamma^{a} \lambda_{+}\right) \\
\quad+\bar{F}_{v b}\left(\bar{\lambda}_{-} \Gamma^{v b} \lambda_{+}\right)+\bar{F}_{\nu}\left(\bar{\lambda}_{-} \Gamma^{\nu} \Gamma_{11} \lambda_{+}\right)+\bar{F}_{v a b c} \\
\\
\left.+\bar{\lambda}_{-} \Gamma^{v a b c} \lambda_{+}\right)+\bar{F}^{v a b}\left(\bar{\lambda}_{+} \Gamma_{v a b} \Gamma_{11} \lambda_{-}\right) \\
\left.\quad+\bar{F}_{f g l m n}\left(\bar{\lambda}_{+} \Gamma^{f g l m n} \lambda_{-}\right)+\bar{F}_{l m n}\left(\bar{\lambda}_{-} \Gamma^{l m n} \lambda_{+}\right)\right] \quad(1.39)
\end{aligned}
$$

where now $\epsilon_{012}=1, i=1,2 / \mu, \nu, \rho=0,1,2 / a, b, c, d$, $f, g, l, m, n=3, \ldots, 9$; and the ansatz $\lambda_{-}=P_{-} \lambda$ was used. The expressions for $F_{\mu}, F_{a}, F_{a b}, F_{v a b d f}, \bar{F}, \bar{F}_{a}, \bar{F}_{\nu}, \bar{F}_{v b}$, $\bar{F}_{\text {vabc }}, \bar{F}_{v a b}, \bar{F}_{f g l m}, \bar{F}_{l m n}$ are given in Appendix B. These expressions are different from zero, so the following constraints guarantee the nilpotency of the BRST charge:

$\partial_{i} E^{i}=0 \quad$ (Gauss law),

$$
\begin{array}{ll}
\bar{\lambda}_{+} \Gamma_{\mu} \lambda_{+}=0, & \bar{\lambda}_{+} \Gamma_{\mu \nu} \lambda_{+}=0, \\
\bar{\lambda}_{-} \Gamma_{\mu} \lambda_{-}=0, & \bar{\lambda}_{-} \Gamma_{a} \Gamma_{11} \lambda_{-}=0, \\
\bar{\lambda}_{-} \Gamma_{a b} \lambda_{-}=0, & \bar{\lambda}_{-} \Gamma_{\mu a b c d} \lambda_{-}=0, \\
\bar{\lambda}_{-} \Gamma_{11} \lambda_{+}=0, & \bar{\lambda}_{-} \Gamma_{a} \lambda_{+}=0, \\
\bar{\lambda}_{-} \Gamma_{\mu a} \lambda_{+}=0, & \bar{\lambda}_{-} \Gamma_{\mu} \Gamma_{11} \lambda_{+}=0, \\
\bar{\lambda}_{-} \Gamma_{\mu a b c} \lambda_{+}=0, & \bar{\lambda}_{+} \Gamma_{\mu a b} \Gamma_{11} \lambda_{-}=0, \\
\bar{\lambda}_{+} \Gamma_{f g l m n} \lambda_{-}=0, & \bar{\lambda}_{-} \Gamma_{l m n} \lambda_{+}=0
\end{array}
$$

with the ansatz $\lambda_{-}=P_{-} \lambda,(i=1,2 / \mu, v=0,1,2 / a, b, c$, $d, f, g, l, m, n=3, . ., 9)$. 


\section{Solving the constraints}

In order to solve the constraints (1.40) we use the methodology used in $[21,22]$. The Dirac matrices $\Gamma^{m}(m=0,1, . ., 9)$ are combined into five creation operators $a^{i}(i=1, \ldots, 5)$ and five annihilation operators $a_{i}(i=1, \ldots, 5)$ as follows:

$$
\begin{array}{llrl}
a^{1} & =\frac{1}{2}\left(\Gamma^{1}-i \Gamma^{2}\right), & a^{2}=\frac{1}{2}\left(\Gamma^{3}-i \Gamma^{4}\right), \\
a^{3} & =\frac{1}{2}\left(\Gamma^{5}-i \Gamma^{6}\right), & & \\
a^{4} & =\frac{1}{2}\left(\Gamma^{7}-i \Gamma^{8}\right), & a^{5} & =\frac{1}{2}\left(\Gamma^{9}+\Gamma^{0}\right), \\
a_{1} & =\frac{1}{2}\left(\Gamma^{1}+i \Gamma^{2}\right), & a_{2} & =\frac{1}{2}\left(\Gamma^{3}+i \Gamma^{4}\right), \\
a_{3} & =\frac{1}{2}\left(\Gamma^{5}+i \Gamma^{6}\right), & \\
a_{4}=\frac{1}{2}\left(\Gamma^{7}+i \Gamma^{8}\right), & a_{5}=\frac{1}{2}\left(\Gamma^{9}-\Gamma^{0}\right)
\end{array}
$$

The following identities hold:

$$
\left\{a_{i}, a^{j}\right\}=\delta_{i j},\left\{a^{i}, a^{j}\right\}=0, \quad\left\{\Gamma_{11}, a^{i}\right\}=0,
$$

$\left\{\Gamma_{11}, a_{j}\right\}=0, \quad a_{i}=a^{i \dagger}$,

$$
\text { with now } i, j=1, . ., 5 \text {. }
$$

Here we introduce the vacuum state $\mid 0>$ and the state $<0$ | with the equations:

$a_{i}|0>=0, \quad<0| a^{i}=0, \quad i=1, . ., 5$.

We decompose a 32-component Dirac spinor into a sum of a positive chirality 16-component part and a negative chirality 16-component part as follows:

$$
\begin{aligned}
\mid \lambda>= & \left(\lambda_{+}\left|0>+\frac{1}{2} \lambda_{i j} a^{j} a^{i}\right| 0>\right. \\
& \left.+\frac{1}{4 !} \lambda^{i} \epsilon_{i j k l m} a^{j} a^{k} a^{l} a^{m} \mid 0>\right) \\
& +\left(\frac{1}{5 !} \lambda_{+}^{\prime} \epsilon_{i j k l m} a^{i} a^{j} a^{k} a^{l} a^{m} \mid 0>\right. \\
& \left.+\frac{1}{3 !} \lambda^{i j} \epsilon_{i j k l m} a^{k} a^{l} a^{m}\left|0>+\lambda_{i}^{\prime} a^{i}\right| 0>\right) \\
= & (1+\overline{10}+5)+(1+10+\overline{5}),
\end{aligned}
$$

where $\lambda_{i j}=-\lambda_{j i}, \lambda^{\prime i j}=-\lambda^{\prime j i}$.

Let us try to solve from (1.40) the constraints $\bar{\lambda}_{+} \Gamma_{\mu} \lambda_{+}=$ 0 and $\bar{\lambda}_{+} \Gamma_{\mu \nu} \lambda_{+}=0$ (where $\mu, \nu=0,1,2$ ).

It is interesting to note that $\lambda_{+}$can be written as a sum of two pure spinors $\lambda_{1+}=P_{+} \lambda_{+}$and $\lambda_{2+}=P_{-} \lambda_{+}$where $P_{ \pm}=\frac{1}{2}\left(1 \pm \Gamma_{0} \Gamma_{1} \Gamma_{2}\right)$ and the constraints $\bar{\lambda}_{+} \Gamma_{\mu} \lambda_{+}=0$, $\bar{\lambda}_{+} \Gamma_{\mu \nu} \lambda_{+}=0,(\mu, \nu=0,1,2)$ can be expressed equivalently as two pure spinor constraints: $\bar{\lambda}_{1+} \Gamma_{m} \lambda_{1+}=0$, $\bar{\lambda}_{2+} \Gamma_{m} \lambda_{2+}=0,(m=0, \ldots, 9)$.
The constraints $\bar{\lambda}_{+} \Gamma_{\mu} \lambda_{+}=0$ can be written as follows:

$$
\begin{aligned}
& <\lambda_{+}\left|C a_{1}\right| \lambda_{+}>=0, \\
& <\lambda_{+}\left|C a^{1}\right| \lambda_{+}>=0, \\
& <\lambda_{+}\left|C a_{5}\right| \lambda_{+}>=<\lambda_{+}\left|C a^{5}\right| \lambda_{+}>,
\end{aligned}
$$

whereas the constraints $\bar{\lambda}_{+} \Gamma_{\mu \nu} \lambda_{+}=0,(\mu, \nu=0,1,2)$ can be expressed as:

$$
\begin{aligned}
& <\lambda_{+}\left|C\left(a^{5}-a_{5}\right)\left(a^{1}+a_{1}\right)\right| \lambda_{+}>=0, \\
& <\lambda_{+}\left|C\left(a^{1}+a_{1}\right)\left(a^{1}-a_{1}\right)\right| \lambda_{+}>0=0, \\
& <\lambda_{+}\left|C\left(a^{5}-a_{5}\right)\left(a^{1}-a_{1}\right)\right| \lambda_{+}>=0 .
\end{aligned}
$$

For a general $\mid \lambda_{+}>$we can write

$$
\begin{aligned}
\mid \lambda_{+}>= & \lambda_{++}\left|0>+\frac{1}{2} \lambda_{+i j} a^{j} a^{i}\right| 0> \\
& +\frac{1}{4 !} \lambda_{+}^{i} \epsilon_{i j k l m} a^{j} a^{k} a^{l} a^{m} \mid 0> \\
& +\lambda_{+i}^{\prime} a^{i}\left|0>+\frac{1}{3 !} \lambda_{+}^{i j} \epsilon_{i j l k m} a^{l} a^{k} a^{m}\right| 0> \\
& +\frac{1}{5 !} \lambda_{++}^{\prime} \epsilon_{i j k l m} a^{i} a^{j} a^{k} a^{l} a^{m} \mid 0>,
\end{aligned}
$$

where now $\epsilon_{12345}=1$.

Thus we have the following relations:

$$
\begin{aligned}
& <\lambda_{+}\left|C a^{1}\right| \lambda_{+}> \\
& =2\left(\lambda_{++} \lambda_{+}^{1}+\frac{1}{8} \epsilon_{1 i j k l} \lambda_{+i j} \lambda_{+l k}\right)-4 \lambda_{+i}^{\prime} \lambda_{+}^{\prime i 1}=0, \\
& <\lambda_{+}\left|C a_{1}\right| \lambda_{+}> \\
& =2 \lambda^{i} \lambda_{+i 1}-2 \lambda_{+1}^{\prime} \lambda_{++}^{\prime}+\epsilon_{1 i j i^{\prime} j^{\prime}} \lambda_{+}^{\prime i j} \lambda_{+}^{\prime^{\prime} j^{\prime}}=0, \\
& <\lambda_{+}\left|C a^{5}\right| \lambda_{+}>=<\lambda_{+}\left|C a_{5}\right| \lambda_{+}> \\
& \Rightarrow 2\left(\lambda_{++} \lambda_{+}^{5}+\frac{1}{8} \epsilon_{5 i j k l} \lambda_{+i j} \lambda_{+l k}\right)-4 \lambda_{+i}^{\prime} \lambda_{+}^{\prime i 5} \\
& =2 \lambda_{+}^{i} \lambda_{+i 5}-2 \lambda_{+5}^{\prime} \lambda_{++}^{\prime}+\epsilon_{5 i j i^{\prime} j^{\prime}} \lambda_{+}^{\prime i j} \lambda_{+}^{\prime i^{\prime} j^{\prime}} .
\end{aligned}
$$

On the other hand the constraints $\bar{\lambda}_{+} \Gamma^{\mu v} \lambda_{+}=0$ can be rewritten as follows:

$$
\begin{aligned}
\frac{1}{2}< & \lambda_{+}\left|C\left(a_{1} a^{1}-a^{1} a_{1}\right)\right| \lambda_{+}> \\
= & -\lambda_{++} \lambda_{++}^{\prime}+4 \lambda_{+i 1} \lambda_{+}^{\prime i 1}-\lambda_{+i j} \lambda^{\prime i j} \\
& +\lambda_{+}^{i} \lambda_{+i}^{\prime}-2 \lambda_{+}^{1} \lambda_{+1}^{\prime}=0, \\
< & \lambda_{+}\left|C\left(a^{5}-a_{5}\right) a^{1}\right| \lambda_{+}> \\
= & 4 \lambda_{++} \lambda_{+}^{\prime 51}+4 \lambda_{+}^{\prime i 1} \lambda_{+i 5}+2 \lambda_{+5}^{\prime} \lambda_{+}^{1} \\
& -\epsilon_{15 i j i} \lambda_{+i j} \lambda_{+i^{\prime}}^{\prime}=0, \\
\frac{1}{2}< & \lambda_{+}\left|C\left(a^{5}-a_{5}\right) a_{1}\right| \lambda_{+}> \\
= & -2 \lambda_{+i 1} \lambda_{+}^{\prime i 5}+\lambda_{+51} \lambda_{++}^{\prime}+\lambda_{+}^{5} \lambda_{+1}^{\prime} \\
& -\epsilon_{i j k 15} \lambda_{+}^{\prime i j} \lambda_{+}^{k}=0 .
\end{aligned}
$$


From (2.8)-(2.11) we obtain the non-trivial solution

$$
\begin{aligned}
\lambda_{+}^{1}= & \frac{1}{\lambda_{++}}\left(2 \lambda_{+i}^{\prime} \lambda_{+}^{\prime i 1}+\frac{1}{8} \epsilon_{1 i j k l} \lambda_{+i j} \lambda_{+k l}\right), \\
\lambda_{+1}^{\prime}= & \frac{1}{2 \lambda_{++}^{\prime}}\left(2 \lambda_{+}^{i} \lambda_{+i 1}+\epsilon_{1 i j k l} \lambda_{+}^{\prime i j} \lambda_{+}^{\prime k l}\right), \\
\lambda_{+}^{5}= & \frac{1}{\lambda_{++}}\left(\lambda_{+}^{i} \lambda_{+i 5}-\lambda_{+5}^{\prime} \lambda_{++}^{\prime}+2 \lambda_{+i}^{\prime} \lambda_{+}^{\prime i 5}\right. \\
& +\frac{1}{2} \epsilon_{5 i j k l} \lambda_{+}^{\prime i j} \lambda_{+}^{\prime}{ }_{+} l \\
\lambda_{+}= & \frac{1}{4 \lambda_{++}}\left(-4 \lambda_{+}^{\prime i 1} \lambda_{+i 5}-2 \lambda_{+i j}^{\prime} \lambda_{+k l} \lambda_{+}^{1}+\epsilon_{15 i j k} \lambda_{+i j} \lambda_{+k}^{\prime}\right), \\
\lambda_{+51}^{\prime}= & \frac{1}{\lambda_{++}^{\prime}}\left(2 \lambda_{+i 1} \lambda_{+}^{\prime i 5}-\lambda_{+}^{5} \lambda_{+1}^{\prime}+\epsilon_{15 i j k} \lambda^{\prime i j} \lambda_{+}^{k}\right), \\
\lambda_{++}^{\prime}= & \frac{1}{\lambda_{++}}\left(4 \lambda_{+i 1} \lambda_{+}^{\prime i 1}-\lambda_{+i j} \lambda_{+}^{\prime i j}+\lambda_{+}^{i} \lambda_{+i}^{\prime}-2 \lambda_{+}^{1} \lambda_{+1}^{\prime}\right) .
\end{aligned}
$$

Now from (1.40) we deal with the following constraints:

$\bar{\lambda}_{-} \Gamma^{\mu} \lambda_{-}=0, \quad \bar{\lambda}_{-} \Gamma^{a} \Gamma_{11} \lambda_{-}=0$,

$\bar{\lambda}_{-} \Gamma^{a b} \lambda_{-}=0, \quad \bar{\lambda}_{-} \Gamma^{\mu a b c d} \lambda_{-}=0$,

where $\mu=0,1,2 / a, b, c, d=3, . ., 9$ with $\lambda_{-}=P_{-} \lambda\left(P_{-}\right.$ is given in (1.38)). Thus, we can write

$P_{-}=\frac{1}{2}\left[1+i\left(a^{5}-a_{5}\right)\left(a_{1} a^{1}-a^{1} a_{1}\right)\right]$.

We have that $P_{-}^{T} C=C P_{-}$where $C$ is the charge conjugation matrix. So for example the constraints $\bar{\lambda}_{-} \Gamma^{\mu} \lambda_{-}=0$ can be rewritten as follows:

$<\lambda\left|C P_{-} a^{1} P_{-}\right| \lambda>=0$,

$<\lambda\left|C P_{-} a_{1} P_{-}\right| \lambda>=0$,

$<\lambda\left|C P_{-} a^{5} P_{-}\right| \lambda>=<\lambda\left|C P_{-} a_{5} P_{-}\right| \lambda>$.

The solution of the constraints in (2.13) is the trivial one. This can be shown as follows. Let us consider the following 16 constraints:

$\bar{\lambda}_{-} \Gamma^{13579} \lambda_{-}=0, \quad \bar{\lambda}_{-} \Gamma^{13589} \lambda_{-}=0, \quad \bar{\lambda}_{-} \Gamma^{13679} \lambda_{-}=0$,

$\bar{\lambda}_{-} \Gamma^{13689} \lambda_{-}=0$

$\bar{\lambda}_{-} \Gamma^{14579} \lambda_{-}=0, \quad \bar{\lambda}_{-} \Gamma^{14679} \lambda_{-}=0, \quad \bar{\lambda}_{-} \Gamma^{14589} \lambda_{-}=0$,

$\bar{\lambda}_{-} \Gamma^{14689} \lambda_{-}=0$

$\bar{\lambda}_{-} \Gamma^{23579} \lambda_{-}=0, \quad \bar{\lambda}_{-} \Gamma^{23589} \lambda_{-}=0, \quad \bar{\lambda}_{-} \Gamma^{23679} \lambda_{-}=0$,

$\bar{\lambda}_{-} \Gamma^{23689} \lambda_{-}=0$,

$\bar{\lambda}_{-} \Gamma^{24579} \lambda_{-}=0, \quad \bar{\lambda}_{-} \Gamma^{24589} \lambda_{-}=0, \quad \bar{\lambda}_{-} \Gamma^{24679} \lambda_{-}=0$,

$\bar{\lambda}_{-} \Gamma^{24689} \lambda_{-}=0$.

which can be rewritten as:

$$
\begin{aligned}
& <\lambda\left|C a^{1}\left(\left(a^{5}+a_{5}\right)-i\left(a_{5} a^{5}-a^{5} a_{5}\right)\right) a^{2} a^{3} a^{4}\right| \lambda> \\
& \quad=-\left(\lambda_{+}\right)^{2}+\left(\lambda_{5}^{\prime}\right)^{2}+2 i\left(\lambda_{+} \lambda_{5}^{\prime}\right)=0
\end{aligned}
$$

$$
\begin{aligned}
& <\lambda\left|C a^{1}\left(\left(a^{5}+a_{5}\right)-i\left(a_{5} a^{5}-a^{5} a_{5}\right)\right) a^{2} a^{3} a_{4}\right| \lambda> \\
& =-\left(\lambda_{45}\right)^{2}+\left(\lambda_{4}^{\prime}\right)^{2}-2 i\left(\lambda_{4}^{\prime} \lambda_{45}\right)=0 \\
& <\lambda\left|C a^{1}\left(\left(a^{5}+a_{5}\right)-i\left(a_{5} a^{5}-a^{5} a_{5}\right)\right) a^{2} a_{3} a^{4}\right| \lambda> \\
& =-\left(\lambda_{35}\right)^{2}+\left(\lambda_{3}^{\prime}\right)^{2}-2 i\left(\lambda_{3}^{\prime} \lambda_{35}\right)=0 \\
& <\lambda\left|C a^{1}\left(\left(a^{5}+a_{5}\right)-i\left(a_{5} a^{5}-a^{5} a_{5}\right)\right) a_{2} a^{3} a^{4}\right| \lambda> \\
& =-\left(\lambda_{52}\right)^{2}+\left(\lambda_{2}^{\prime}\right)^{2}-2 i\left(\lambda_{2}^{\prime} \lambda_{52}\right)=0 \\
& <\lambda\left|C a^{1}\left(\left(a^{5}+a_{5}\right)-i\left(a_{5} a^{5}-a^{5} a_{5}\right)\right) a^{2} a_{3} a_{4}\right| \lambda> \\
& =-\left(\lambda_{34}\right)^{2}+4\left(\lambda^{\prime 12}\right)^{2}-4 i\left(\lambda_{34} \lambda^{\prime 12}\right)=0 \\
& <\lambda\left|C a^{1}\left(\left(a^{5}+a_{5}\right)-i\left(a_{5} a^{5}-a^{5} a_{5}\right)\right) a_{2} a^{3} a_{4}\right| \lambda> \\
& =-\left(\lambda_{42}\right)^{2}+4\left(\lambda^{\prime 13}\right)^{2}-4 i\left(\lambda_{42} \lambda^{\prime 13}\right)=0 \\
& <\lambda\left|C a^{1}\left(\left(a^{5}+a_{5}\right)-i\left(a_{5} a^{5}-a^{5} a_{5}\right)\right) a_{2} a_{3} a^{4}\right| \lambda> \\
& =-\left(\lambda_{23}\right)^{2}+4\left(\lambda^{\prime 14}\right)^{2}-4 i\left(\lambda_{23} \lambda^{\prime 14}\right)=0 \\
& <\lambda\left|C a^{1}\left(\left(a^{5}+a_{5}\right)-i\left(a_{5} a^{5}-a^{5} a_{5}\right)\right) a_{2} a_{3} a_{4}\right| \lambda> \\
& =-\left(\lambda^{1}\right)^{2}+4\left(\lambda^{\prime 15}\right)^{2}-4 i\left(\lambda^{1} \lambda^{\prime 15}\right)=0 \\
& <\lambda\left|C a_{1}\left(\left(a^{5}+a_{5}\right)-i\left(a_{5} a^{5}-a^{5} a_{5}\right)\right) a^{2} a^{3} a^{4}\right| \lambda> \\
& =-\left(\lambda_{15}\right)^{2}+\left(\lambda_{1}^{\prime}\right)^{2}+2 i\left(\lambda_{1}^{\prime} \lambda_{15}\right)=0 \\
& <\lambda\left|C a_{1}\left(\left(a^{5}+a_{5}\right)-i\left(a_{5} a^{5}-a^{5} a_{5}\right)\right) a^{2} a^{3} a_{4}\right| \lambda> \\
& =-\left(\lambda_{14}\right)^{2}+4\left(\lambda^{\prime 23}\right)^{2}+4 i\left(\lambda_{14} \lambda^{\prime 23}\right)=0 \\
& <\lambda\left|C a_{1}\left(\left(a^{5}+a_{5}\right)-i\left(a_{5} a^{5}-a^{5} a_{5}\right)\right) a^{2} a_{3} a^{4}\right| \lambda> \\
& =-\left(\lambda_{13}\right)^{2}+4\left(\lambda^{\prime 24}\right)^{2}-4 i\left(\lambda_{13} \lambda^{\prime 24}\right)=0 \\
& <\lambda\left|C a_{1}\left(\left(a^{5}+a_{5}\right)-i\left(a_{5} a^{5}-a^{5} a_{5}\right)\right) a_{2} a^{3} a^{4}\right| \lambda> \\
& =-\left(\lambda_{12}\right)^{2}+4\left(\lambda^{\prime 34}\right)^{2}+4 i\left(\lambda_{12} \lambda^{\prime 34}\right)=0 \\
& <\lambda\left|C a_{1}\left(\left(a^{5}+a_{5}\right)-i\left(a_{5} a^{5}-a^{5} a_{5}\right)\right) a^{2} a_{3} a_{4}\right| \lambda> \\
& =-\left(\lambda^{2}\right)^{2}+4\left(\lambda^{\prime 52}\right)^{2}-4 i\left(\lambda^{2} \lambda^{\prime 52}\right)=0 \\
& <\lambda\left|C a_{1}\left(\left(a^{5}+a_{5}\right)-i\left(a_{5} a^{5}-a^{5} a_{5}\right)\right) a_{2} a^{3} a_{4}\right| \lambda> \\
& =-\left(\lambda^{3}\right)^{2}+4\left(\lambda^{\prime} 53\right)^{2}+4 i\left(\lambda^{3} \lambda^{\prime 53}\right)=0 \\
& <\lambda\left|C a_{1}\left(\left(a^{5}+a_{5}\right)-i\left(a_{5} a^{5}-a^{5} a_{5}\right)\right) a_{2} a_{3} a^{4}\right| \lambda> \\
& =-\left(\lambda^{4}\right)^{2}+4\left(\lambda^{\prime 45}\right)^{2}+4 i\left(\lambda^{4} \lambda^{\prime 45}\right)=0 \\
& <\lambda\left|C a_{1}\left(\left(a^{5}+a_{5}\right)-i\left(a_{5} a^{5}-a^{5} a_{5}\right)\right) a_{2} a_{3} a_{4}\right| \lambda> \\
& =-\left(\lambda^{5}\right)^{2}+\left(\lambda_{+}^{\prime}\right)^{2}-2 i\left(\lambda^{5} \lambda_{+}^{\prime}\right)=0
\end{aligned}
$$

and the expression for $\mid \lambda>$ is given in (2.4). According to Comment 2 in $[21,22]$ we choose all the $\lambda \mathrm{s}$ and $\lambda^{\prime} \mathrm{s}$ to be real. However the Dirac spinor becomes complex in a general Lorentz frame. Then the relations (2.17)(2.32) imply that all the components of $\mid \lambda>$ are zero and the solution of the constraints in (2.16) is the trivial one. It is interesting to note that $\lambda_{-}$corresponds to $\theta_{-}$which is a gauge degree of freedom as pointed out in [16].

Thus, the novel nontrivial solution (2.12) found for one of the spinor fields actually proves that the pure spinor formalism can be successfully applied to non-relativistic systems with kappa symmetry as is the case of the IIA D2brane. 


\section{Conclusions}

This paper is the first attempt to extend the application of the pure spinor formalism to non-relativistic systems with kappa symmetry expecting to draw some conclusions for the general case as well. This can very useful especially in cases where it is difficult to solve the pure spinor constraints in the general case. We treated the non-relativistic IIA D2 brane in the framework of this formalism [23] and we derived the fermionic constraints corresponding to the rescaled fermionic coordinates. We introduced two commuting spinor fields each one corresponding to a fermionic coordinate. The nilpotency of the BRST charge leads to a set of constraints for the two spinor fields including pure spinor constraints. Nontrivial solutions are found for the spinor field $\lambda_{+}$which corresponds to the fermionic coordinate $\theta_{+}$. It is interesting to note that this solution can be written as the sum of two pure spinors $\lambda_{1+}=P_{+} \lambda_{+}$and $\lambda_{2+}=P_{-} \lambda_{+}$where $P_{ \pm}=\frac{1}{2}\left(1 \pm \Gamma_{0} \Gamma_{1} \Gamma_{2}\right)$. The solution for the spinor field $\lambda_{-}$corresponding to $\theta_{-}$which, according to the proof given in [16] constitutes a gauge degree of freedom, is the trivial one. So for example the expression of the BRST charge for $\theta_{-}=0$ in the Schrödinger representation is given by $Q=\int d^{2} \sigma\left[-i\left(\lambda_{1+}+\lambda_{2+}\right)^{\alpha} \frac{\partial}{\partial \theta_{+}^{\alpha}}+\right.$ $\left.2 T_{N R} \epsilon_{i j k}\left(\bar{\theta}_{+} \Gamma^{i} \lambda_{1+}\right) \partial_{1} x^{j} \partial_{2} x^{k}\right]$. Cohomological issues concerning the BRST charge are currently under investigation [26-29]. This study can also be performed for more general manifolds and for general dimensions as well. We would like to finally mention that the treatment of the relativistic Dp-brane in the framework of the pure spinor formalism has been reported in [24].

Acknowledgements We gratefully acknowledge support from "CONACYT grant A1-S-38041, Aspectos gravitatorios de la correspondencia holográfica. AHA acknowledges a VIEP-BUAP research grant, he thanks as well SNI and PRODEP for partial financial support, while JEP is grateful to the Instituto de Física y Matemáticas, at UMSNH, in Morelia, for the warm hospitality and the stimulating atmosphere generated during his visit at the Institute.

Data Availability Statement This manuscript has no associated data or the data will not be deposited. [Authors' comment: There is no data to be deposited because our work makes no use of any data.]

Open Access This article is distributed under the terms of the Creative Commons Attribution 4.0 International License (http://creativecomm ons.org/licenses/by/4.0/), which permits unrestricted use, distribution, and reproduction in any medium, provided you give appropriate credit to the original author(s) and the source, provide a link to the Creative Commons license, and indicate if changes were made.

Funded by $\mathrm{SCOAP}^{3}$.

\section{Appendix A}

For the matrices $\Gamma^{m}(m=0, . ., 9)$ we use the Majorana representation $\left(\Gamma^{0}\right.$ is real antisymmetric, $\Gamma^{i}(i=1, \ldots, 9)$ are real symmetric)

$\left\{\Gamma^{m}, \Gamma^{n}\right\}=2 \eta^{m n}$

where $\eta^{m n}=\operatorname{diag}(-1,1, \ldots, 1)$ and $m, n=0, \ldots, 9$; and

$$
C=\Gamma_{0},
$$

$\left(\Gamma^{m}\right)^{T}=-\Gamma_{0} \Gamma^{m} \Gamma_{0}^{-1}$.

A basis for the $32 \times 32$ matrices is given by (see Appendix $\mathrm{B}$ in [25] for instance):

$$
\begin{aligned}
B= & \left\{I, \Gamma^{A} \Gamma^{A B}, \Gamma^{A B C}, \Gamma^{A B C D}, \Gamma^{A B C D E}, \Gamma^{A B C D} \Gamma_{11},\right. \\
& \left.\Gamma^{A B} \Gamma_{11}, \Gamma^{A} \Gamma_{11}, \Gamma_{11}\right\},
\end{aligned}
$$

with $A<B<C<D<E$.

For the differential form $d \theta$ we use the convention given in [18]:

$d \theta=d \sigma^{\mu} \partial_{\mu} \theta=-\partial_{\mu} \theta d \sigma^{\mu}$.

The following identities hold for $\lambda_{-}=P_{-} \lambda$ :

$\Gamma_{\mu \nu} \lambda_{-}=-\epsilon_{\mu \nu \rho} \Gamma^{\rho} \lambda_{-}$

$\Gamma_{\mu \nu \rho} \lambda_{-}=-\epsilon_{\mu \nu \rho} \lambda_{-}$

$\epsilon_{012}=1$.

\section{Appendix B}

$$
\begin{aligned}
F_{\mu}= & 2\left[p_{\mu}-E^{i}\left(\bar{\theta}_{-} \Gamma_{11} \Gamma_{\mu} \partial_{i} \theta_{+}+\bar{\theta}_{+} \Gamma_{11} \Gamma_{\mu} \partial_{i} \theta_{-}\right)\right] \\
& +2 T_{N R} \epsilon_{0 j k}\left[-2\left(\bar{\theta}_{+} \Gamma_{\mu \nu} \partial_{j} \theta_{+}\right) R_{k}^{v}\right. \\
& \left.+\left(\bar{\theta}_{+} \Gamma_{a \mu} \partial_{j} \theta_{-}+\bar{\theta}_{-} \Gamma_{a \mu} \partial_{j} \theta_{+}\right) u_{k}^{a}\right] \\
& +\frac{T_{N R}}{96} \epsilon_{0 j k}\left[( \overline { \theta } _ { - } \Gamma ^ { v } \partial _ { k } \theta _ { - } ) \left(-89\left(\bar{\theta}_{+} \Gamma_{\mu \nu} \partial_{j} \theta_{+}\right)\right.\right. \\
& \left.+22\left(\bar{\theta}_{+} \partial_{j} \theta_{+}\right) \eta_{\mu \nu}\right)+\left(\bar{\theta}_{+} \Gamma^{v} \partial_{k} \theta_{+}\right)\left(83\left(\bar{\theta}_{-} \Gamma_{\mu \nu} \partial_{j} \theta_{-}\right)\right. \\
& \left.+6\left(\bar{\theta}_{-} \partial_{j} \theta_{-}\right) \eta_{\mu \nu}\right)-2\left(\bar{\theta}_{-} \Gamma^{a} \partial_{k} \theta_{+}\right. \\
& \left.+\bar{\theta}_{+} \Gamma^{a} \partial_{k} \theta_{-}\right)\left(47\left(\bar{\theta}_{+} \Gamma_{\mu a} \partial_{j} \theta_{-}\right)+23\left(\bar{\theta}_{-} \Gamma_{\mu a} \partial_{j} \theta_{+}\right)\right) \\
& -5\left(\bar{\theta}_{+} \Gamma_{\mu} \Gamma_{\nu a} \partial_{j} \theta_{-}\right)\left(\partial_{k} \bar{\theta}_{+} \Gamma^{\nu a} \theta_{-}\right) \\
& -\frac{1}{3}\left(\partial_{k} \bar{\theta}_{+} \Gamma^{a} \theta_{-}\right)\left(37\left(\partial_{j} \bar{\theta}_{-} \Gamma_{\mu a} \theta_{+}\right)\right. \\
& \left.-66\left(\partial_{j} \bar{\theta}_{+} \Gamma_{\mu a} \theta_{-}\right)\right)-\left(\partial_{j} \theta_{-} \Gamma^{a} \theta_{+}\right)\left(21\left(\partial_{k} \bar{\theta}_{+} \Gamma_{\mu a} \theta_{-}\right)\right. \\
& \left.-2\left(\partial_{k} \theta_{-} \Gamma_{\mu a} \theta_{+}\right)\right)-2\left(\bar{\theta}_{+} \Gamma_{11} \partial_{-}+\bar{\theta}_{-} \Gamma_{11} \partial \theta_{+}\right) \\
& \times\left(25\left(\bar{\theta}_{+} \Gamma_{11} \Gamma_{\mu} \partial_{j} \theta_{-}\right)+31\left(\bar{\theta}_{-} \Gamma_{11} \Gamma_{\mu} \partial_{j} \theta_{+}\right)\right) \\
& +2\left(\bar{\theta}_{-} \Gamma_{11} \Gamma^{\nu} \partial_{k} \theta_{+}+\bar{\theta}_{+} \Gamma_{11} \Gamma^{\nu} \partial_{k} \theta_{-}\right) \\
& \times\left(\bar{\theta}_{+} \Gamma_{11} \Gamma_{\mu} \Gamma_{\nu} \partial_{j} \theta_{-}+2\left(\bar{\theta}_{-} \Gamma_{11} \partial_{j} \theta_{+}\right) \eta_{\mu \nu}\right) \\
& -\left(\partial_{j} \bar{\theta}_{-} \Gamma_{11} \Gamma^{\nu} \theta_{-}\right)\left(64\left(\partial_{k} \bar{\theta}_{+} \Gamma_{11} \theta_{-}\right)\right.
\end{aligned}
$$




$$
\begin{aligned}
& -11\left(\partial_{k} \bar{\theta}_{+} \Gamma_{11} \Gamma_{\mu \nu} \theta_{-}\right)+46\left(\partial_{k} \bar{\theta}_{-} \Gamma_{11} \theta_{+}\right) \eta_{\mu \nu} \\
& \left.-2\left(\partial_{k} \bar{\theta}_{-} \Gamma_{11} \Gamma_{\nu} \Gamma_{\mu} \theta_{+}\right)\right)+\left(\bar{\theta}_{-} \Gamma_{11} \Gamma_{\nu} \partial_{k} \theta_{+}\right) \\
& \times\left(9\left(\bar{\theta}_{-} \Gamma_{11} \Gamma_{\nu} \Gamma_{\mu} \theta_{+}\right)+34\left(\bar{\theta}_{-} \Gamma_{11} \partial_{j} \theta_{+}\right) \eta_{\mu \nu}\right. \\
& \left.+23\left(\bar{\theta}_{+} \Gamma_{11} \partial_{j} \theta_{-}\right) \eta_{\mu \nu}\right)-7\left(\partial_{j} \bar{\theta}_{-} \Gamma_{11} \Gamma_{a} \theta_{-}\right) \\
& \times\left(\partial_{k} \bar{\theta}_{+} \Gamma_{11} \Gamma_{\mu a} \theta_{+}\right)+5\left(\partial_{k} \bar{\theta}_{+} \Gamma_{11} \Gamma^{v a} \Gamma_{\mu} \theta_{+}\right) \\
& \left(\bar{\theta}_{-} \Gamma_{\nu a} \Gamma_{11} \partial_{j} \theta_{-}\right)+\left(\bar{\theta}_{-} \Gamma^{v a} \Gamma_{11} \Gamma_{\mu} \partial_{j} \theta_{-}\right) \\
& \times\left(\partial_{k} \bar{\theta}_{+} \Gamma_{\nu a} \Gamma_{11} \theta_{+}\right)-\left(\partial_{k} \bar{\theta}_{+} \Gamma_{11} \Gamma^{a} \theta_{+}\right) \\
& \left.\left(\partial_{j} \bar{\theta}_{-} \Gamma_{11} \Gamma_{\mu a} \theta_{-}\right)+\left(\bar{\theta}_{+} \Gamma_{\nu a} \partial_{j} \theta_{-}\right)\left(\bar{\theta}_{-} \Gamma^{\nu a} \Gamma_{\mu} \partial_{k} \theta_{+}\right)\right]
\end{aligned}
$$

$$
\begin{aligned}
F_{a}= & -2 E_{i} u_{a}^{i}+2 T_{N R} \epsilon_{0 j k}\left(\bar{\theta}_{+} \Gamma_{11} \partial_{j} \theta_{-}+\bar{\theta}_{-} \Gamma_{11} \partial_{j} \theta_{+}\right) u_{a k} \\
& +\frac{T_{N R}}{3} \epsilon_{0 j k}\left[-\frac{1}{16}\left(\bar{\theta}_{+} \Gamma^{b} \partial_{j} \theta_{-}\right)\right. \\
& \times\left(10\left(\bar{\theta}_{-} \Gamma_{a b} \Gamma_{11} \partial_{k} \theta_{+}\right)-\left(\bar{\theta}_{+} \Gamma_{a b} \Gamma_{11} \partial_{k} \theta_{-}\right)\right. \\
& \left.+3\left(\partial_{k} \theta_{-} \Gamma_{11} \theta_{+}\right) \eta_{a b}+45\left(\partial_{k} \bar{\theta}_{+} \Gamma_{11} \theta_{-}\right) \eta_{a b}\right) \\
& +\frac{3}{16}\left(\bar{\theta}_{-} \Gamma^{b} \partial_{j} \theta_{+}\right)\left(-8\left(\partial_{k} \bar{\theta}_{+} \Gamma_{a b} \Gamma_{11} \theta_{-}\right)\right. \\
& \left.+12\left(\bar{\theta}_{-} \Gamma_{11} \partial_{k} \theta_{+}\right) \eta_{a b}-\left(\bar{\theta}_{+} \Gamma_{11} \partial_{k} \theta_{-}\right) \eta_{a b}\right) \\
& +\frac{7}{8}\left(\bar{\theta}_{+} \Gamma^{\mu} \partial_{k} \theta_{+}\right)\left(\partial_{j} \bar{\theta}_{-} \Gamma_{\mu a} \Gamma_{11} \theta_{-}\right) \\
& +\frac{1}{48}\left(\bar{\theta}_{+} \Gamma_{11} \Gamma^{\mu} \partial_{k} \theta_{-}\right)\left(5\left(\partial_{j} \bar{\theta}_{+} \Gamma_{\mu a} \theta_{-}\right)-6\left(\partial_{j} \bar{\theta}_{-} \Gamma_{\mu a} \theta_{+}\right)\right) \\
& -\frac{1}{16}\left(\partial_{j} \bar{\theta}_{-} \Gamma_{11} \Gamma^{b} \theta_{-}\right)\left(13\left(\partial_{k} \bar{\theta}_{+} \Gamma_{a b} \theta_{+}\right)\right. \\
& \left.+3\left(\bar{\theta}_{+} \partial_{k} \theta_{+}\right) \eta_{a b}\right)-\frac{1}{32}\left(\partial_{j} \bar{\theta}_{-} \Gamma_{a b c} \theta_{+}\right) \\
& \times\left(2\left(\partial_{k} \bar{\theta}_{+} \Gamma^{b c} \Gamma_{11} \theta_{-}\right)+\partial_{k} \bar{\theta}_{-} \Gamma^{b c} \Gamma_{11} \theta_{+}\right) \\
& +\frac{1}{16}\left(\partial_{j} \bar{\theta}_{-} \Gamma^{\mu b} \theta_{+}\right)\left(-2\left(\partial_{k} \bar{\theta}_{-} \Gamma_{\mu a b} \Gamma_{11} \theta_{+}\right)\right. \\
& -6\left(\partial_{k} \bar{\theta}_{+} \Gamma_{11} \Gamma_{\mu a b} \theta_{-}\right)+9\left(\bar{\theta}_{-} \Gamma_{11} \Gamma_{\mu} \partial_{k} \theta_{+}\right) \eta_{a b} \\
& \left.+\left(\bar{\theta}_{+} \Gamma_{11} \Gamma_{\mu} \partial_{k} \theta_{-}\right) \eta_{a b}\right)-\frac{9}{16}\left(\bar{\theta}_{-} \Gamma^{\mu} \partial_{j} \theta_{-}\right) \\
& \times\left(\bar{\theta}_{+} \Gamma_{\mu a} \Gamma_{11} \partial_{k} \theta_{+}\right)+\frac{3}{8}\left(\bar{\theta}_{+} \Gamma^{\mu b} \Gamma_{11} \partial_{k} \theta_{+}\right)\left(\partial_{j} \bar{\theta}_{-} \Gamma_{\mu a b} \theta_{-}\right) \\
& +\frac{117}{64}\left(\bar{\theta}_{-} \partial_{j} \theta_{-}\right)\left(\bar{\theta}_{+} \Gamma_{11} \Gamma_{a} \partial_{k} \theta_{+}\right) \\
& +\frac{137}{64}\left(\bar{\theta}_{-} \Gamma_{a b} \partial_{j} \theta_{-}\right)\left(\bar{\theta}_{+} \Gamma_{11} \Gamma^{b} \partial_{k} \theta_{+}\right) \\
& +\frac{1}{16}\left(\bar{\theta}_{-} \Gamma^{\mu b} \partial_{j} \theta_{+}\right)\left(\partial_{k} \bar{\theta}_{-} \Gamma_{11} \Gamma_{\mu a b} \theta_{+}\right) \\
& -\frac{1}{16}\left(\bar{\theta}_{-} \Gamma_{11} \Gamma^{\mu b} \partial_{j} \theta_{-}\right) \\
& \left.\times\left(\partial_{k} \bar{\theta}_{+} \Gamma_{\mu a b} \theta_{+}\right)-\frac{1}{16}\left(\bar{\theta}_{-} \Gamma^{b c} \partial_{k} \theta_{-}\right)\left(\bar{\theta}_{+} \Gamma_{a b c} \Gamma_{11} \partial_{j} \theta_{+}\right)\right] \\
&
\end{aligned}
$$

$$
\begin{aligned}
F_{a b}= & T_{N R} \epsilon_{0 j k} u_{a j} u_{b k}+\frac{T_{N R}}{16} \epsilon_{0 j k}\left[\left(\bar{\theta}_{+} \Gamma^{d} \partial_{j} \theta_{-}\right)\right. \\
& \times\left(-\frac{14}{3}\left(\partial_{k} \bar{\theta}_{-} \Gamma_{a b} \Gamma_{d} \theta_{+}\right)\right. \\
& +\frac{14}{3}\left(\partial_{k} \bar{\theta}_{-} \Gamma_{d} \Gamma_{a b} \theta_{+}\right)+\frac{35}{6}\left(\partial_{k} \bar{\theta}_{+} \Gamma_{a b} \Gamma_{d} \theta_{-}\right) \\
& -\frac{15}{2}\left(\partial_{k} \bar{\theta}_{+} \Gamma_{d} \Gamma_{a b} \theta_{-}\right)+\frac{16}{3}\left(\bar{\theta}_{+} \Gamma_{b} \partial_{k} \theta_{-}\right) \eta_{a d} \\
& \left.+\frac{32}{3}\left(\bar{\theta}_{-} \Gamma_{b} \partial_{k} \theta_{+}\right) \eta_{a d}\right)+\frac{1}{6}\left(\bar{\theta}_{-} \Gamma^{d} \partial_{j} \theta_{+}\right)\left(7\left(\partial_{k} \bar{\theta}_{+} \Gamma_{d} \Gamma_{a b} \theta_{-}\right)\right.
\end{aligned}
$$

$+35\left(\partial_{k} \bar{\theta}_{+} \Gamma_{a b} \Gamma_{d} \theta_{-}\right)$

$+4\left(\partial_{k} \bar{\theta}_{-} \Gamma_{d} \Gamma_{a b} \theta_{+}\right)-33\left(\partial_{k} \bar{\theta}_{-} \Gamma_{a b} \Gamma_{d} \theta_{+}\right)$

$\left.-32\left(\bar{\theta}-\Gamma_{a} \partial_{k} \theta_{+}\right) \eta_{b d}\right)+\frac{1}{6}\left(\bar{\theta}_{-} \Gamma_{11} \Gamma^{\mu} \partial_{k} \theta_{+}\right)$

$\times\left(-4\left(\partial_{j} \bar{\theta}_{+} \Gamma_{11} \Gamma_{\mu a b} \theta_{-}\right)+37\left(\partial_{j} \bar{\theta}_{-} \Gamma_{11} \Gamma_{\mu a b} \theta_{-}\right)\right)$

$+\frac{15}{4}\left(\bar{\theta}_{+} \Gamma^{\mu d} \Gamma_{11} \partial_{k} \theta_{+}\right)\left(\partial_{j} \bar{\theta}_{-} \Gamma_{11} \Gamma_{\mu d} \Gamma_{a b} \theta_{-}\right)$

$+\frac{25}{12}\left(\partial_{j} \bar{\theta}_{-} \Gamma^{\mu d} \theta_{+}\right)\left(\partial_{k} \bar{\theta}_{+} \Gamma_{\mu d} \Gamma_{a b} \theta_{-}\right)$

$-\frac{77}{12}\left(\bar{\theta}_{+} \Gamma^{d} \Gamma_{11} \partial_{k} \theta_{+}\right)\left(\partial_{j} \bar{\theta}_{-} \Gamma_{11} \Gamma_{d} \Gamma_{a b} \theta_{-}\right)$

$-\frac{35}{4}\left(\partial_{j} \bar{\theta}_{-} \Gamma_{11} \theta_{+}\right)\left(\partial_{k} \bar{\theta}_{+} \Gamma_{11} \Gamma_{a b} \theta_{-}\right)$

$-\frac{45}{4}\left(\bar{\theta}_{-} \Gamma_{a b} \partial_{j} \theta_{-}\right)\left(\bar{\theta}_{+} \partial_{k} \theta_{+}\right)+\frac{37}{4}\left(\bar{\theta}_{+} \Gamma^{\mu} \partial_{k} \theta_{+}\right)$

$\times\left(\partial_{j} \bar{\theta}_{-} \Gamma_{\mu a b} \theta_{-}\right)+\frac{1}{6}\left(\bar{\theta}_{-} \Gamma_{11} \Gamma^{d} \partial_{j} \theta_{-}\right)\left(5\left(\partial_{k} \bar{\theta}_{+} \Gamma_{11} \Gamma_{a b} \Gamma_{d} \theta_{+}\right)\right.$

$\left.+39\left(\partial_{k} \bar{\theta}_{+} \Gamma_{d} \Gamma_{a b} \Gamma_{11} \theta_{+}\right)\right)-3\left(\bar{\theta}_{-} \Gamma_{11} \partial_{k} \theta_{+}\right)\left(\partial_{j} \bar{\theta}_{-} \Gamma_{11} \Gamma_{a b} \theta_{+}\right)$

$+\left(\bar{\theta}_{-} \Gamma^{\mu d} \partial_{j} \theta_{+}\right)\left(\partial_{k} \bar{\theta}_{-} \Gamma_{a b} \Gamma_{\mu d} \theta_{+}\right)-\left(\bar{\theta}_{-} \Gamma_{d f} \partial_{k} \theta_{-}\right)$

$\times\left(\bar{\theta}_{+} \Gamma^{d} \Gamma_{a b} \Gamma^{f} \partial_{j} \theta_{+}\right)-\frac{1}{4}\left(\bar{\theta}_{+} \Gamma_{11} \Gamma^{\mu} \partial_{k} \theta_{-}\right)\left(\partial_{j} \bar{\theta}_{+} \Gamma_{11} \Gamma_{\mu a b} \theta_{-}\right)$

$-\frac{1}{2}\left(\bar{\theta}_{-} \partial_{j} \theta_{-}\right)\left(\partial_{k} \bar{\theta}_{+} \Gamma_{a b} \theta_{+}\right)$

$-\frac{1}{6}\left(\bar{\theta}_{-} \Gamma_{11} \Gamma^{\mu d} \partial_{j} \theta_{-}\right)\left(\partial_{k} \bar{\theta}_{+} \Gamma_{11} \Gamma_{\mu a b} \Gamma_{d} \theta_{+}\right)$

$+\frac{1}{6}\left(\partial_{k} \bar{\theta}_{+} \Gamma_{d f} \Gamma_{11} \theta_{-}\right)\left(\partial_{j} \bar{\theta}_{-} \Gamma_{11} \Gamma^{d} \Gamma_{a b} \Gamma^{f} \theta_{+}\right)$

$\left.-4\left(\bar{\theta}_{-} \Gamma^{\mu} \partial_{j} \theta_{-}\right)\left(\partial_{k} \bar{\theta}_{+} \Gamma_{\mu a b} \theta_{+}\right)\right]$

$\bar{F}=2 T_{N R} \epsilon_{0 j k}\left[\widetilde{\mathcal{F}}_{j k}^{(1)}-\frac{1}{3}\left(\left(\bar{\theta}_{-} \Gamma^{\mu} \partial_{k} \theta_{-}\right)\left(\bar{\theta}_{+} \Gamma_{\mu} \Gamma_{11} \partial_{j} \theta_{-}\right.\right.\right.$

$\left.+\bar{\theta}_{-} \Gamma_{\mu} \Gamma_{11} \partial_{j} \theta_{+}\right)$

$\left.\left.+\left(\bar{\theta}_{-} \Gamma^{a} \Gamma_{11} \partial_{j} \theta_{-}\right)\left(\bar{\theta}_{-} \Gamma_{a} \partial_{k} \theta_{+}+\bar{\theta}_{+} \Gamma_{a} \partial_{k} \theta_{-}\right)\right)\right]$

$\bar{F}_{\nu}=-4 E^{i} R_{\nu i}+4 T_{N R} \epsilon_{0 j k} R_{\nu k}\left(\bar{\theta}_{+} \Gamma_{11} \partial_{j} \theta_{-}+\bar{\theta}-\Gamma_{11} \partial_{j} \theta_{+}\right)$

$+\frac{1}{48} T_{N R} \epsilon_{0 j k}\left[-\left(\bar{\theta}_{-} \Gamma^{\mu a} \partial_{j} \theta_{+}\right)\right.$

$\times\left(16\left(\partial_{k} \bar{\theta}_{-} \Gamma_{11} \Gamma_{a} \theta_{-}\right) \eta_{\mu \nu}-5\left(\partial_{k} \bar{\theta}_{-} \Gamma_{\mu \nu a} \Gamma_{11} \theta_{-}\right)\right)$

$-12\left(\partial_{k} \bar{\theta}_{-} \Gamma_{11} \Gamma^{a} \theta_{-}\right)\left(\bar{\theta}_{+} \Gamma_{v a} \partial_{j} \theta_{-}\right)$

$-\left(\bar{\theta}_{-} \Gamma^{\mu} \partial_{j} \theta_{-}\right)\left(-37\left(\partial_{k} \bar{\theta}_{+} \Gamma_{\mu \nu} \Gamma_{11} \theta_{-}\right)\right.$

$\left.+41\left(\partial_{k} \bar{\theta}_{+} \Gamma_{11} \theta_{-}\right) \eta_{\mu \nu}+18\left(\partial_{k} \bar{\theta}_{-} \Gamma_{11} \theta_{+}\right) \eta_{\mu \nu}\right)$

$+\left(\partial_{j} \bar{\theta}_{-} \Gamma_{v a} \Gamma_{b} \theta_{-}\right)\left(-5\left(\partial_{k} \bar{\theta}_{+} \Gamma^{b} \Gamma^{a} \Gamma_{11} \theta_{-}\right)\right.$

$\left.+2\left(\partial_{k} \bar{\theta}_{-} \Gamma^{b} \Gamma^{a} \Gamma_{11} \theta_{+}\right)\right)-\left(\bar{\theta}_{-} \Gamma^{a b} \partial_{k} \theta_{-}\right)$

$\times\left(7\left(\bar{\theta}_{-} \Gamma_{v a b} \Gamma_{11} \partial_{j} \theta_{+}\right)+2\left(\bar{\theta}_{+} \Gamma_{v a b} \Gamma_{11} \partial_{j} \theta_{-}\right)\right)$

$+\left(\partial_{j} \bar{\theta}_{+} \Gamma_{11} \Gamma^{\mu} \theta_{-}\right)\left(70\left(\bar{\theta}_{-} \partial_{k} \theta_{-}\right) \eta_{\mu \nu}\right.$

$\left.+59\left(\partial_{k} \bar{\theta}_{-} \Gamma_{\mu \nu} \theta_{-}\right)\right)+\left(\partial_{j} \bar{\theta}_{-} \Gamma_{\nu a} \Gamma_{11} \theta_{-}\right)$

$\times\left(49\left(\bar{\theta}_{-} \Gamma^{a} \partial_{k} \theta_{+}\right)+24\left(\bar{\theta}_{+} \Gamma^{a} \partial_{k} \theta_{-}\right)\right)$

$\left.-5\left(\partial_{k} \bar{\theta}_{+} \Gamma^{\mu a} \Gamma_{\nu} \theta_{-}\right)\left(\bar{\theta}_{-} \Gamma_{11} \Gamma_{\mu a} \partial_{j} \theta_{-}\right)\right]$

$\bar{F}_{a}=4\left(p_{a}-E^{i}\left(\bar{\theta}_{-} \Gamma_{11} \Gamma_{a} \partial_{i} \theta_{-}\right)\right)$

$-2 T_{N R} \epsilon_{0 j k}\left[\left(\bar{\theta}_{-} \Gamma_{\mu a} \partial_{k} \theta_{+}+\bar{\theta}_{+} \Gamma_{\mu a} \partial_{k} \theta_{-}\right) R_{j}^{\mu}\right.$ 


$$
\begin{aligned}
& \left.-\left(\bar{\theta}_{-} \Gamma_{a b} \partial_{k} \theta_{-}\right) u_{j}^{b}\right]+\frac{2}{3} T_{N R} \epsilon_{0 j k}\left[\frac{1}{16}\left(\bar{\theta}_{-} \Gamma^{\mu} \partial_{j} \theta_{-}\right)\right. \\
& \times\left(25\left(\partial_{k} \bar{\theta}_{+} \Gamma_{\mu a} \theta_{-}\right)+19\left(\partial_{k} \theta_{-} \Gamma_{\mu a} \theta_{+}\right)\right) \\
& +\frac{1}{8}\left(\bar{\theta}_{-} \partial_{j} \theta_{-}\right)\left(21\left(\partial_{k} \bar{\theta}_{+} \Gamma_{a} \theta_{-}\right)+9\left(\partial_{k} \bar{\theta}_{-} \Gamma_{a} \theta_{+}\right)\right) \\
& -\frac{1}{32}\left(\partial_{j} \bar{\theta}_{-} \Gamma_{\mu a b} \theta_{-}\right)\left(5\left(\bar{\theta}_{-} \Gamma^{\mu b} \partial_{k} \theta_{+}\right)\right. \\
& \left.-2\left(\bar{\theta}_{+} \Gamma^{\mu b} \partial_{k} \theta_{-}\right)\right)+\frac{1}{32}\left(\bar{\theta}_{-} \Gamma_{a b} \partial_{k} \theta_{-}\right)\left(7\left(\bar{\theta}_{-} \Gamma^{b} \partial_{j} \theta_{+}\right)\right. \\
& \left.+12\left(\bar{\theta}_{+} \Gamma^{b} \partial_{j} \theta_{-}\right)\right)+\frac{1}{16}\left(\partial_{j} \bar{\theta}_{-} \Gamma_{11} \Gamma_{\mu a} \theta_{-}\right) \\
& \times\left(3\left(\partial_{k} \bar{\theta}_{-} \Gamma_{11} \Gamma^{\mu} \theta_{+}\right)+10\left(\partial_{k} \bar{\theta}_{+} \Gamma_{11} \Gamma^{\mu} \theta_{-}\right)\right) \\
& +\frac{1}{16}\left(\bar{\theta}_{-} \Gamma_{a} \Gamma_{11} \partial_{j} \theta_{-}\right)\left(29\left(\bar{\theta}_{+} \Gamma_{11} \partial_{k} \theta_{-}\right)\right. \\
& \left.+20\left(\bar{\theta}_{-} \Gamma_{11} \partial_{k} \theta_{+}\right)\right)+\frac{1}{32}\left(\bar{\theta}_{-} \Gamma_{11} \Gamma^{b} \partial_{k} \theta_{-}\right) \\
& \times\left(2\left(\bar{\theta}_{+} \Gamma_{11} \Gamma_{a b} \partial_{j} \theta_{-}\right)-\bar{\theta}_{-} \Gamma_{11} \Gamma^{a b} \partial_{j} \theta_{+}\right) \\
& +\frac{1}{32}\left(\partial_{j} \bar{\theta}_{-} \Gamma_{11} \Gamma_{a b c} \theta_{-}\right)\left(\bar{\theta}_{+} \Gamma_{11} \Gamma^{b c} \partial_{k} \theta_{-}\right. \\
& \left.-5\left(\bar{\theta}_{-} \Gamma_{11} \Gamma^{b c} \partial_{k} \theta_{+}\right)\right)+\frac{1}{32}\left(\bar{\theta}_{-} \Gamma^{b c} \partial_{j} \theta_{-}\right) \\
& \times\left(7\left(\partial_{k} \bar{\theta}_{+} \Gamma_{a b c} \theta_{-}\right)+2\left(\partial_{k} \bar{\theta}_{-} \Gamma_{a b c} \theta_{+}\right)\right) \\
& \left.-\frac{5}{32}\left(\partial_{k} \bar{\theta}_{+} \Gamma_{11} \Gamma_{\mu a b} \theta_{-}\right)\left(\bar{\theta}_{-} \Gamma_{11} \Gamma^{\mu b} \partial_{j} \theta_{-}\right)\right] \\
& \bar{F}_{v a}=4 T_{N R} \epsilon_{0 j k} R_{v j} u_{a k}+\frac{1}{48} T_{N R} \epsilon_{0 j k}\left[\left(\bar{\theta}_{-} \Gamma^{\mu} \partial_{j} \theta_{+}\right)\right. \\
& \times\left(37\left(\bar{\theta}_{-} \Gamma_{a} \partial_{k} \theta_{+}\right) \eta_{\mu \nu}\right. \\
& +34\left(\bar{\theta}_{+} \Gamma_{a} \partial_{k} \theta_{-}\right) \eta_{\mu \nu}-5\left(\bar{\theta}_{-} \Gamma_{\mu \nu a} \partial_{k} \theta_{+}\right) \\
& \left.+6\left(\bar{\theta}_{+} \Gamma_{\mu \nu a} \partial_{k} \theta_{-}\right)\right)+\left(\bar{\theta}_{-} \Gamma_{11} \Gamma^{b} \partial_{j} \theta_{-}\right) \\
& \times\left(5\left(\partial_{k} \bar{\theta}_{+} \Gamma_{11} \Gamma_{v}\left(\eta_{a b}+\Gamma_{b a}\right) \theta_{-}\right)\right. \\
& \left.-2\left(\partial_{k} \bar{\theta}_{-} \Gamma_{11} \Gamma_{v b a} \theta_{+}\right)\right)-\left(\bar{\theta}_{-} \Gamma^{b} \partial_{k} \theta_{+}\right) \\
& \times\left(-19\left(\partial_{j} \bar{\theta}_{-} \Gamma_{v b a} \theta_{-}\right)+9\left(\partial_{j} \bar{\theta}_{-} \Gamma_{\nu} \theta_{-}\right) \eta_{a b}\right) \\
& +\left(\bar{\theta}_{-} \Gamma^{b \mu} \Gamma_{11} \partial_{j} \theta_{-}\right)\left(\partial_{k} \bar{\theta}_{+} \Gamma_{\mu} \Gamma_{11} \Gamma_{\nu a} \Gamma_{b} \theta_{-}\right) \\
& +5\left(\bar{\theta}_{-} \Gamma^{\mu} \Gamma_{11} \partial_{k} \theta_{+}\right)\left(\partial_{j} \bar{\theta}_{-} \Gamma_{\mu} \Gamma_{11} \Gamma_{\nu a} \theta_{-}\right) \\
& -\left(\bar{\theta}_{-} \Gamma^{b c} \Gamma_{11} \partial_{k} \theta_{+}\right)\left(\partial_{j} \bar{\theta}_{-} \Gamma_{11} \Gamma_{\nu a b c} \theta_{-}\right) \\
& +\left(\partial_{k} \bar{\theta}_{+} \Gamma^{b \mu_{\theta_{-}}}\right)\left(\partial_{j} \bar{\theta}_{-} \Gamma_{\mu} \Gamma_{\nu a} \Gamma_{b} \theta_{-}\right) \\
& -5\left(\bar{\theta}_{-} \Gamma_{11} \partial_{k} \theta_{+}\right)\left(\bar{\theta}_{-} \Gamma_{\nu a} \Gamma_{11} \partial_{j} \theta_{-}\right)-22\left(\bar{\theta}_{+} \Gamma^{b} \partial_{j} \theta_{-}\right) \\
& \times\left(\bar{\theta}_{-} \Gamma_{v b a} \partial_{k} \theta_{-}\right)+2\left(\bar{\theta}_{+} \Gamma_{11} \Gamma^{\mu} \partial_{k} \theta_{-}\right)\left(-\partial_{j} \bar{\theta}_{-} \Gamma_{11} \Gamma_{\mu v a} \theta_{-}\right. \\
& \left.+2\left(\partial_{j} \bar{\theta}_{-} \Gamma_{11} \Gamma_{a} \theta_{-}\right) \eta_{\mu \nu}\right) \\
& +2\left(\partial_{k} \bar{\theta}_{-} \Gamma^{b \mu} \theta_{+}\right)\left(2\left(\partial_{j} \bar{\theta}_{-} \Gamma_{\mu \nu} \theta_{-}\right) \eta_{a b}+\left(\partial_{j} \bar{\theta}_{-} \Gamma_{\mu \nu b a} \theta_{-}\right)\right. \\
& -2\left(\partial_{j} \bar{\theta}_{-} \Gamma_{b a} \theta_{-}\right) \eta_{\mu \nu} \\
& \left.-\left(\partial_{j} \bar{\theta}-\theta_{-}\right) \eta_{\mu \nu} \eta_{a b}\right)-2\left(\partial_{j} \bar{\theta}_{-} \Gamma_{11} \theta_{+}\right)\left(\partial_{k} \bar{\theta}_{-} \Gamma_{11} \Gamma_{\nu a} \theta_{-}\right) \\
& +\left(\partial_{k} \bar{\theta}_{-} \Gamma^{b c} \theta_{-}\right) \\
& \left.\times\left(\partial_{j} \bar{\theta}_{-} \Gamma_{v a b c} \theta_{+}-2\left(\partial_{j} \bar{\theta}_{+} \Gamma_{\nu a b c} \theta_{-}\right)\right)\right] \\
& \bar{F}^{v a b}=\frac{1}{96} T_{N R} \epsilon_{0 j k}\left[\left(\partial_{k} \bar{\theta}_{-} \Gamma^{\mu} \Gamma_{11} \Gamma^{v a b} \Gamma^{d} \theta_{-}\right)\right. \\
& \times\left(4\left(\partial_{j} \bar{\theta}_{-} \Gamma_{d \mu} \theta_{+}\right)-\partial_{j} \bar{\theta}_{+} \Gamma_{d \mu} \theta_{-}\right) \\
& -6\left(\partial_{k} \bar{\theta}_{-} \Gamma^{d} \theta_{+}\right)\left(\partial_{j} \bar{\theta}_{-}\left(\Gamma_{d} \Gamma^{v a b}+3 \Gamma^{v a b} \Gamma_{d}\right) \Gamma_{11} \theta_{-}\right)
\end{aligned}
$$




$$
\begin{aligned}
& +\left(\partial_{j} \bar{\theta}_{-} \Gamma^{f} \Gamma^{a b c d} \Gamma^{e} \theta_{-}\right)\left(\partial_{k} \bar{\theta}_{+} \Gamma_{f e} \Gamma_{11} \theta_{-}\right. \\
& \left.+2\left(\partial_{k} \bar{\theta}_{-} \Gamma_{f e} \Gamma_{11} \theta_{+}\right)\right)-5\left(\bar{\theta}_{-} \Gamma^{a b c d} \partial_{j} \theta_{-}\right) \\
& \times\left(\bar{\theta}_{-} \Gamma_{11} \partial_{k} \theta_{+}\right)-\left(\bar{\theta}_{-} \Gamma_{e f} \partial_{k} \theta_{-}\right)\left(\bar{\theta}_{-} \Gamma^{e} \Gamma^{a b c d} \Gamma^{f} \partial_{j} \theta_{+}\right. \\
& \left.+2\left(\bar{\theta}_{+} \Gamma^{e} \Gamma^{a b c d} \Gamma^{f} \Gamma_{11} \partial_{j} \theta_{-}\right)\right) \\
& \left.+3\left(\partial_{j} \bar{\theta}_{-} \Gamma^{\mu a b c d} \theta_{-}\right)\left(\partial_{k} \bar{\theta}_{+} \Gamma_{11} \Gamma_{\mu} \theta_{-}\right)\right] \\
& \bar{F}_{\nu a b c}=\frac{1}{288} T_{N R} \epsilon_{0 j k}\left[( \overline { \theta } _ { - } \Gamma ^ { \mu } \partial _ { j } \theta _ { - } ) \left(3\left(\bar{\theta}_{-} \Gamma_{\mu \nu a b c} \partial_{k} \theta_{+}\right)\right.\right. \\
& \left.-23\left(\bar{\theta}-\Gamma_{a b c} \partial_{k} \theta_{+}\right) \eta_{\mu \nu}\right) \\
& +\left(\bar{\theta}_{-} \Gamma^{d} \partial_{j} \theta_{+}\right)\left(5\left(\bar{\theta}_{-} \Gamma_{\nu a b c d} \partial_{k} \theta_{-}\right)\right. \\
& \left.+3\left(\bar{\theta}_{-} \Gamma_{\nu b c} \partial_{k} \theta_{-}\right) \eta_{a d}\right)-24\left(\bar{\theta}_{+} \Gamma_{a} \partial_{j} \theta_{-}\right) \\
& \times\left(\bar{\theta}_{-} \Gamma_{v b c} \partial_{k} \theta_{-}\right)+\left(\bar{\theta}_{-} \Gamma_{11} \Gamma^{\mu} \partial_{k} \theta_{+}\right) \\
& \times\left(-3\left(\bar{\theta}_{-} \Gamma_{11} \Gamma_{a b c} \partial_{j} \theta_{-}\right) \eta_{\mu \nu}+5\left(\partial_{j} \bar{\theta}_{-} \Gamma_{11} \Gamma_{\mu \nu a b c} \theta_{-}\right)\right) \\
& +\left(\bar{\theta}_{-} \Gamma_{11} \Gamma^{d} \partial_{j} \theta_{-}\right)\left(\bar{\theta}_{-} \Gamma_{11} \Gamma_{\nu a b c} \Gamma_{d} \partial_{k} \theta_{+}\right. \\
& -4\left(\bar{\theta}_{-} \Gamma_{d} \Gamma_{v a b c} \Gamma_{11} \partial_{k} \theta_{+}\right) \\
& \left.+12\left(\bar{\theta}_{+} \Gamma_{v b c} \Gamma_{11} \partial_{k} \theta_{-}\right) \eta_{a d}\right)+\left(\bar{\theta}_{-} \Gamma^{\mu d} \partial_{k} \theta_{+}\right) \\
& \times\left(\partial_{j} \bar{\theta}_{-} \Gamma_{\mu} \Gamma_{\nu a b c} \Gamma_{d} \theta_{-}-4\left(\partial_{j} \bar{\theta}_{-} \Gamma_{d} \Gamma_{\nu a b c} \Gamma_{\mu} \theta_{-}\right)\right) \\
& -\left(\bar{\theta}_{-} \Gamma^{d f} \partial_{k} \theta_{-}\right)\left(\bar{\theta}_{-} \Gamma_{d} \Gamma_{v a b c} \Gamma_{f} \partial_{j} \theta_{+}\right. \\
& \left.+2\left(\bar{\theta}_{+} \Gamma_{d} \Gamma_{v a b c} \Gamma_{f} \partial_{j} \theta_{-}\right)\right)+\left(\partial_{k} \bar{\theta}_{-} \Gamma_{d} \Gamma_{11} \Gamma_{v a b c} \Gamma_{f} \theta_{-}\right) \\
& \times\left(3\left(\partial_{j} \bar{\theta}_{+} \Gamma^{d f} \Gamma_{11} \theta_{-}\right)+2\left(\partial_{j} \bar{\theta}_{-} \Gamma^{d f} \Gamma_{11} \theta_{+}\right)\right) \\
& +5\left(\partial_{j} \bar{\theta}_{+} \Gamma_{11} \theta_{-}\right)\left(\partial_{k} \bar{\theta}-\Gamma_{11} \Gamma_{v a b c} \theta_{-}\right) \\
& +3\left(\bar{\theta}_{-} \Gamma_{11} \Gamma_{\mu d} \partial_{j} \theta_{-}\right)\left(\partial_{k} \bar{\theta}_{+} \Gamma^{\mu} \Gamma_{11} \Gamma_{\nu a b c} \Gamma^{d} \theta_{-}\right) \\
& \left.-3\left(\bar{\theta}_{-} \partial_{j} \theta_{-}\right)\left(\partial_{k} \bar{\theta}_{+} \Gamma_{v a b c} \theta_{-}\right)\right]
\end{aligned}
$$

$F^{v a b d f}=\frac{1}{96} T_{N R} \epsilon_{0 j k}\left[6\left(\bar{\theta}_{-} \Gamma^{c} \partial_{j} \theta_{+}\right)\left(\bar{\theta}_{-} \Gamma^{\nu a b d f} \Gamma_{c} \partial_{k} \theta_{+}\right.\right.$

$\left.-\bar{\theta}_{-} \Gamma_{c} \Gamma^{v a b d f} \partial_{k} \theta_{+}\right)$

$-2\left(\bar{\theta}_{-} \Gamma_{11} \Gamma_{\mu} \partial_{k} \theta_{+}\right)\left(\partial_{j} \bar{\theta}_{+} \Gamma_{11} \Gamma^{\mu} \Gamma^{v a b d f_{\theta_{-}}}\right.$

$\left.+\partial_{j} \bar{\theta}_{+} \Gamma_{11} \Gamma^{v a b d f} \Gamma^{\mu} \theta_{-}\right)-2\left(\bar{\theta}_{-} \Gamma_{11} \Gamma_{\rho \sigma} \partial_{k} \theta_{+}\right)$

$\times\left(\partial_{j} \bar{\theta}_{+} \Gamma_{11} \Gamma^{\rho} \Gamma^{v a b d f} \Gamma^{\sigma} \theta_{-}\right)+2\left(\bar{\theta}_{-} \Gamma^{c} \partial_{j} \theta_{+}\right)$

$\times\left(\partial_{k} \bar{\theta}_{-} \Gamma^{\nu a d f} \Gamma_{c} \theta_{+}+\partial_{k} \bar{\theta}_{-} \Gamma_{c} \Gamma^{v a b d f} \theta_{+}\right)$

$+3\left(\bar{\theta}_{+} \Gamma^{c} \partial_{j} \theta_{-}\right)\left(-2\left(\partial_{k} \bar{\theta}_{+} \Gamma^{v a b d f} \Gamma_{c} \theta_{-}\right)\right.$

$\left.+\bar{\theta}_{-} \Gamma^{v a b d f} \Gamma_{c} \partial_{k} \theta_{+}\right)-4\left(\bar{\theta}_{-} \Gamma_{11} \Gamma_{\mu} \partial_{k} \theta_{+}\right)$

$\times\left(\partial_{j} \bar{\theta}_{-} \Gamma_{11} \Gamma^{\mu} \Gamma^{v a b d f} \theta_{+}\right)$

$+\left(\bar{\theta}_{+} \Gamma_{11} \Gamma_{\mu} \partial_{k} \theta_{-}\right)\left(\partial_{j} \bar{\theta}_{+} \Gamma_{11} \Gamma^{\mu} \Gamma^{v a b d f_{\theta_{-}}}\right.$

$\left.-2\left(\partial_{j} \bar{\theta}_{+} \Gamma_{11} \Gamma^{v a b d f} \Gamma^{\mu} \theta_{-}\right)\right)$

$-2\left(\partial_{j} \bar{\theta}_{-} \Gamma^{\mu} \theta_{-}\right)\left(\partial_{k} \bar{\theta}_{+} \Gamma_{\mu} \Gamma^{v a b d f} \theta_{+}\right)$

$+2\left(\partial_{k} \bar{\theta}_{+} \Gamma_{\mu c} \theta_{-}\right)\left(\bar{\theta}_{+} \Gamma^{c} \Gamma^{v a b d f} \Gamma^{\mu} \partial_{j} \theta_{-}\right)$

$+2\left(\bar{\theta}_{-} \Gamma^{c} \Gamma_{11} \partial_{j} \theta_{-}\right)\left(\partial_{k} \bar{\theta}_{+} \Gamma_{11} \Gamma^{v a b d f} \Gamma_{c} \theta_{+}\right.$

$\left.-2\left(\partial_{k} \bar{\theta}_{+} \Gamma_{11} \Gamma_{c} \Gamma^{v a b d f} \theta_{+}\right)\right)$

$+2\left(\partial_{k} \bar{\theta}_{+} \Gamma_{11} \theta_{-}\right)\left(\bar{\theta}_{+} \Gamma_{11} \Gamma^{v a b d f} \partial_{j} \theta_{-}\right)+\left(\bar{\theta}_{+} \Gamma^{\mu} \partial_{k} \theta_{+}\right)$

$\times\left(6\left(\partial_{j} \bar{\theta}_{-} \Gamma_{\mu} \Gamma^{v a b d f} \theta_{-}\right)+5\left(\partial_{j} \theta_{-} \Gamma^{v a b d f} \Gamma_{\mu} \theta_{-}\right)\right)$

$+7\left(\bar{\theta}_{+} \Gamma_{\mu \sigma} \partial_{k} \theta_{+}\right)\left(\partial_{j} \bar{\theta}_{-} \Gamma^{\sigma} \Gamma^{v a b d f} \Gamma^{\mu} \theta_{-}\right)$

$-3\left(\bar{\theta}_{+} \Gamma_{\mu c} \partial_{j} \theta_{-}\right)\left(\partial_{k} \bar{\theta}_{+} \Gamma^{c} \Gamma^{v a b d f} \Gamma^{\mu} \theta_{-}\right)$

$-4\left(\bar{\theta}_{+} \Gamma_{11} \partial_{j} \theta_{-}\right)\left(\partial_{k} \bar{\theta}_{+} \Gamma_{11} \Gamma^{v a b d f_{\theta_{-}}}\right)$

$$
\begin{aligned}
& +\left(\partial_{k} \bar{\theta}_{+} \Gamma_{\mu c} \Gamma_{11} \theta_{+}\right)\left(\partial_{j} \bar{\theta}_{-} \Gamma^{c} \Gamma_{11} \Gamma^{\nu a b d f} \Gamma^{\mu} \theta_{-}\right) \\
& +\left(\partial_{k} \bar{\theta}_{+} \Gamma_{\mu} \Gamma_{\sigma} \theta_{+}\right) \\
& \times\left(\partial_{j} \bar{\theta}_{-} \Gamma^{\sigma} \Gamma^{v a b d f} \Gamma^{\mu} \theta_{-}\right)+\left(\bar{\theta}_{+} \Gamma_{11} \Gamma_{\sigma} \Gamma_{\mu} \partial_{j} \theta_{-}\right) \\
& \times\left(\partial_{k} \bar{\theta}_{+} \Gamma^{\sigma} \Gamma_{11} \Gamma^{v a b d f} \Gamma^{\mu} \theta_{-}\right) \\
& -\left(\partial_{k} \bar{\theta}_{+} \Gamma_{11} \Gamma_{c} \theta_{+}\right)\left(\partial_{j} \bar{\theta}_{-} \Gamma_{11} \Gamma^{c} \Gamma^{v a b d f} \theta_{-}\right) \\
& +2\left(\bar{\theta}_{-} \Gamma_{c e} \partial_{k} \theta_{-}\right)\left(\bar{\theta}_{+} \Gamma^{c} \Gamma^{v a b d f} \Gamma^{e} \partial_{j} \theta_{+}\right) \\
& \left.-\frac{1}{4 !} \epsilon_{c g e a b d f} \delta_{c g e}^{[l m n}\right] \frac{T_{N R}}{576}\left[-36\left(\bar{\theta}-\Gamma^{l} \partial_{j} \theta_{+}\right)\right. \\
& \times\left(\partial_{k} \bar{\theta}_{+} \Gamma^{\nu m n} \Gamma_{11} \theta_{-}\right)+2\left(\bar{\theta}_{-} \Gamma^{r} \partial_{j} \theta_{+}\right) \\
& \times\left(\partial_{k} \bar{\theta}_{-} \Gamma_{11} \Gamma^{v l m n} \Gamma_{r} \theta_{+}+\partial_{k} \bar{\theta}_{-} \Gamma_{11} \Gamma_{r} \Gamma^{v l m n} \theta_{+}\right) \\
& +3\left(\bar{\theta}_{+} \Gamma^{r} \partial_{j} \theta_{-}\right) \\
& \times\left(\partial_{k} \bar{\theta}_{+} \Gamma_{r} \Gamma^{v l m n} \Gamma_{11} \theta_{-}+2\left(\partial_{k} \bar{\theta}_{+} \Gamma_{11} \Gamma^{\nu l m n} \Gamma_{r} \theta_{-}\right)\right) \\
& -4\left(\bar{\theta}_{-} \Gamma_{11} \Gamma_{\mu} \partial_{k} \theta_{+}\right) \\
& \times\left(\partial_{j} \bar{\theta}_{-} \Gamma^{\mu} \Gamma^{\nu l m n} \theta_{+}\right)-\left(\bar{\theta}_{+} \Gamma_{11} \Gamma_{\mu} \partial_{k} \theta_{-}\right) \\
& \times\left(2\left(\partial_{j} \bar{\theta}_{+} \Gamma^{v l m n} \Gamma^{\mu} \theta_{-}\right)+\partial_{j} \bar{\theta}_{+} \Gamma^{\mu} \Gamma^{v l m n} \theta_{-}\right) \\
& +2\left(\bar{\theta}_{-} \Gamma^{\mu} \partial_{j} \theta_{-}\right)\left(\partial_{k} \bar{\theta}_{+} \Gamma_{11} \Gamma_{\mu} \Gamma^{\nu l m n} \theta_{+}\right) \\
& -2\left(\partial_{k} \bar{\theta}_{+} \Gamma_{r \mu} \theta_{-}\right)\left(\bar{\theta}_{+} \Gamma^{r} \Gamma^{v l m n} \Gamma_{11} \Gamma^{\mu} \partial_{j} \theta_{-}\right) \\
& +2\left(\bar{\theta}_{-} \Gamma^{r} \Gamma_{11} \partial_{j} \theta_{-}\right)\left(-2\left(\partial_{k} \bar{\theta}_{+} \Gamma_{r} \Gamma^{v l m n} \theta_{+}\right)\right. \\
& \left.+\partial_{k} \bar{\theta}_{+} \Gamma^{\nu l m n} \Gamma_{r} \theta_{+}\right)+2\left(\partial_{k} \bar{\theta}_{+} \Gamma_{11} \theta_{-}\right) \\
& \times\left(\bar{\theta}_{+} \Gamma^{\nu l m n} \partial_{j} \theta_{-}\right)+6\left(\bar{\theta}_{+} \Gamma_{\sigma \mu} \partial_{k} \theta_{+}\right) \\
& \times\left(\partial_{j} \bar{\theta}_{-} \Gamma^{\mu} \Gamma^{\nu l m n} \Gamma_{11} \Gamma^{\sigma} \theta_{-}\right)-\left(\partial_{k} \bar{\theta}_{+} \theta_{+}\right) \\
& \times\left(\partial_{j} \bar{\theta}_{-} \Gamma^{\nu l m n} \Gamma_{11} \theta_{-}\right)+\left(\partial_{k} \bar{\theta}_{+} \Gamma_{\mu r} \Gamma_{11} \theta_{+}\right) \\
& \left(\partial_{j} \bar{\theta}-\Gamma^{r} \Gamma^{v l m n} \Gamma^{\mu} \theta_{-}\right)+\left(\partial_{j} \bar{\theta}_{-} \Gamma_{11} \Gamma_{\mu \sigma} \theta_{+}\right) \\
& \times\left(\partial_{k} \bar{\theta}_{+} \Gamma^{\mu} \Gamma^{v l m n} \Gamma^{\sigma} \theta_{-}\right)+3\left(\partial_{j} \bar{\theta}_{-} \Gamma_{11} \theta_{+}\right) \\
& \times\left(\partial_{k} \bar{\theta}_{+} \Gamma^{v l m n} \theta_{-}\right)+\left(\partial_{k} \bar{\theta}_{+} \Gamma_{11} \Gamma^{r} \theta_{+}\right) \\
& \times\left(\partial_{j} \bar{\theta}-\Gamma_{r} \Gamma^{v l m n} \theta_{-}\right) \\
& +3\left(\bar{\theta}_{+} \Gamma_{r \sigma} \partial_{j} \theta_{-}\right)\left(\partial_{k} \bar{\theta}_{+} \Gamma^{r} \Gamma^{\nu l m n} \Gamma_{11} \Gamma^{\sigma} \theta_{-}\right) \\
& +\left(\bar{\theta}_{+} \Gamma^{\mu} \partial_{k} \theta_{+}\right)\left(6\left(\partial_{j} \bar{\theta}_{-} \Gamma_{\mu} \Gamma^{\nu l m n} \Gamma_{11} \theta_{-}\right)\right. \\
& \left.+5\left(\partial_{j} \bar{\theta}_{-} \Gamma^{\nu l m n} \Gamma_{11} \Gamma_{\mu} \theta_{-}\right)\right)+2\left(\bar{\theta}_{-} \Gamma_{r s} \partial_{k} \theta_{-}\right) \\
& \left.\times\left(\bar{\theta}_{+} \Gamma^{r} \Gamma^{\nu l m n} \Gamma_{11} \Gamma^{s} \partial_{j} \theta_{+}\right)\right]
\end{aligned}
$$

$\bar{F}_{f g l m n}=\frac{1}{96 \times 5 !} T_{N R} \epsilon_{0 j k}\left[3\left(\bar{\theta}_{-} \Gamma^{\mu} \partial_{j} \theta_{-}\right)\left(-5\left(\bar{\theta}_{-} \Gamma_{\mu f g l m n} \partial_{k} \theta_{+}\right)\right.\right.$

$\left.+4\left(\bar{\theta}_{+} \Gamma_{\mu f g l m n} \partial_{k} \theta_{-}\right)\right)+\left(\bar{\theta}_{-} \Gamma^{a} \partial_{j} \theta_{+}\right)$

$\times\left(17\left(\bar{\theta}_{-} \Gamma_{f g l m n} \Gamma_{a} \partial_{k} \theta_{-}\right)-22\left(\bar{\theta}_{-} \Gamma_{a} \Gamma_{f g l m n} \partial_{k} \theta_{-}\right)\right)$

$+4\left(\bar{\theta}_{+} \Gamma^{a} \partial_{j} \theta_{-}\right)\left(\bar{\theta}_{-} \Gamma_{f g l m n} \Gamma_{a} \partial_{k} \theta_{-}\right.$

$\left.-5\left(\bar{\theta}_{-} \Gamma_{a} \Gamma_{f g l m n} \partial_{k} \theta_{-}\right)\right)+9\left(\bar{\theta}_{-} \Gamma_{11} \Gamma^{\mu} \partial_{k} \theta_{+}\right)$

$\times\left(\partial_{j} \bar{\theta}_{-} \Gamma_{11} \Gamma_{\mu f g l m n} \theta_{-}\right)$

$+12\left(\bar{\theta}_{+} \Gamma_{11} \Gamma^{\mu} \partial_{k} \theta_{-}\right)\left(\partial_{j} \bar{\theta}_{-} \Gamma_{\mu f g l m n} \Gamma_{11} \theta_{-}\right)$

$+\left(\partial_{j} \bar{\theta}_{-} \Gamma_{11} \Gamma^{a} \theta_{-}\right)$

$\times\left(-4\left(\partial_{k} \bar{\theta}_{+} \Gamma_{11} \Gamma_{f g l m n} \Gamma_{a} \theta_{-}\right)\right.$

$+13\left(\partial_{k} \bar{\theta}_{+} \Gamma_{11} \Gamma_{a} \Gamma_{f g l m n} \theta_{-}\right)+6\left(\partial_{k} \bar{\theta}_{-} \Gamma_{11} \Gamma_{a} \Gamma_{f g l m n} \theta_{+}\right)$

$\left.+2\left(\partial_{k} \bar{\theta}_{-} \Gamma_{11} \Gamma_{f g l m n} \Gamma_{a} \theta_{+}\right)\right)$

$-\left(\partial_{k} \bar{\theta}_{-} \Gamma_{11} \Gamma^{a} \Gamma_{f g l m n} \Gamma^{b} \theta_{-}\right)\left(4\left(\partial_{j} \bar{\theta}_{-} \Gamma_{b} \Gamma_{a} \Gamma_{11} \theta_{+}\right)\right.$

$\left.+3\left(\partial_{j} \bar{\theta}_{+} \Gamma_{b} \Gamma_{a} \Gamma_{11} \theta_{-}\right)\right)+3\left(\bar{\theta}_{-} \Gamma_{b} \Gamma_{a} \partial_{j} \theta_{-}\right)$ 


$$
\begin{aligned}
& \times\left(\partial_{k} \bar{\theta}_{+} \Gamma^{b} \Gamma_{f g l m n} \Gamma^{a} \theta_{-}\right)+2\left(\bar{\theta}_{-} \Gamma^{a b} \partial_{k} \theta_{-}\right) \\
& \times\left(\bar{\theta}_{-} \Gamma_{a} \Gamma_{f g l m n} \Gamma_{b} \partial_{j} \theta_{+}+\bar{\theta}_{+} \Gamma_{a} \Gamma_{f g l m n} \Gamma_{b} \partial_{j} \theta_{-}\right) \\
& -6\left(\bar{\theta}_{-} \Gamma_{11} \partial_{k} \theta_{+}\right)\left(\partial_{j} \bar{\theta}_{-} \Gamma_{11} \Gamma_{f g l m n} \theta_{-}\right) \\
& +12\left(\partial_{j} \bar{\theta}_{-} \Gamma_{11} \theta_{+}\right)\left(\partial_{k} \bar{\theta}_{-} \Gamma_{11} \Gamma_{f g l m n} \theta_{-}\right) \\
& -3\left(\partial_{k} \bar{\theta}_{+} \Gamma_{11} \Gamma_{f g l m n} \Gamma^{\mu a} \theta_{-}\right)\left(\bar{\theta}_{-} \Gamma_{11} \Gamma_{\mu a} \partial_{j} \theta_{-}\right) \\
& -\left(\bar{\theta}_{-} \Gamma^{\mu a} \partial_{k} \theta_{+}\right)\left(4\left(\partial_{j} \bar{\theta}_{-} \Gamma_{\mu a} \Gamma_{f g l m n} \theta_{-}\right)\right. \\
& \left.-\partial_{j} \bar{\theta}_{-} \Gamma_{f g l m n} \Gamma_{\mu a} \theta_{-}\right)-2\left(\bar{\theta}_{+} \Gamma^{\mu a} \partial_{k} \theta_{-}\right) \\
& \left.\times\left(\bar{\theta}_{-} \Gamma_{\mu a} \Gamma_{f g l m n} \partial_{j} \theta_{-}+\partial_{j} \Gamma_{\mu a} \Gamma_{f g l m n} \theta_{-}\right)\right] \\
& -\frac{1}{192 \times 5 !} \epsilon_{0 j k} \epsilon_{d e f g l m n} \delta_{d e}^{b a}\left[\left(16\left(\bar{\theta}_{+} \Gamma_{c} \partial_{j} \theta_{-}\right)\right.\right. \\
& \left.+21\left(\bar{\theta}_{-} \Gamma_{c} \partial_{j} \theta_{+}\right)\right)\left(\bar{\theta}_{-} \Gamma^{a b c} \Gamma_{11} \partial_{k} \theta_{-}\right) \\
& +8\left(7\left(\bar{\theta}_{+} \Gamma^{a} \partial_{j} \theta_{-}\right)+3\left(\bar{\theta}_{-} \Gamma^{a} \partial_{j} \theta_{+}\right)\right)\left(\bar{\theta}_{-} \Gamma^{b} \Gamma_{11} \partial_{k} \theta_{-}\right) \\
& -\left(\bar{\theta}_{-} \Gamma_{\mu} \partial_{j} \theta_{-}\right) \\
& \times\left(12\left(\bar{\theta}_{+} \Gamma^{\mu a b} \Gamma_{11} \partial_{k} \theta_{-}\right)+11\left(\bar{\theta}_{-} \Gamma_{11} \Gamma^{\mu a b} \partial_{k} \theta_{+}\right)\right) \\
& +8\left(\bar{\theta}_{-} \Gamma_{\mu} \Gamma^{b} \Gamma_{11} \partial_{j} \theta_{-}\right)\left(\bar{\theta}_{+} \Gamma^{\mu a} \partial_{k} \theta_{-}\right. \\
& \left.+\bar{\theta}_{-} \Gamma^{\mu a} \partial_{k} \theta_{+}\right)+\left(\partial_{k} \bar{\theta}_{+} \Gamma_{\mu c} \theta_{-}\right)\left(\partial_{j} \bar{\theta}_{-} \Gamma^{\mu a b c} \Gamma_{11} \theta_{-}\right. \\
& \left.+2\left(\bar{\theta}_{-} \Gamma^{\mu b} \Gamma_{11} \partial_{j} \theta_{-}\right) \eta^{a c}\right) \\
& +\left(\bar{\theta}_{-} \Gamma_{11} \Gamma_{c} \partial_{k} \theta_{-}\right)\left(-8\left(\bar{\theta}_{+} \Gamma^{a b c} \partial_{j} \theta_{-}\right)\right. \\
& \left.+3\left(\bar{\theta}_{-} \Gamma^{a b c} \partial_{j} \theta_{+}\right)\right)+\left(\bar{\theta}_{-} \Gamma^{\mu a b} \partial_{j} \theta_{-}\right) \\
& \times\left(12\left(\bar{\theta}_{+} \Gamma_{11} \Gamma_{\mu} \partial_{k} \theta_{-}\right)-17\left(\bar{\theta}_{-} \Gamma_{11} \Gamma_{\mu} \partial_{k} \theta_{+}\right)\right) \\
& -4\left(\partial_{j} \bar{\theta}_{-} \Gamma_{c d} \Gamma_{11} \theta_{+}\right)\left(\partial_{k} \bar{\theta}_{-} \Gamma^{c} \Gamma^{a b} \Gamma^{d} \theta_{-}\right) \\
& +\left(\partial_{j} \bar{\theta}_{+} \Gamma_{c d} \Gamma_{11} \theta_{-}\right)\left(2\left(\partial_{k} \bar{\theta}_{-} \Gamma^{c d} \Gamma^{a b} \theta_{-}\right)\right. \\
& \left.-17\left(\bar{\theta}_{-} \partial_{k} \theta_{-}\right) \eta^{a c} \eta^{b d}\right)+\left(\bar{\theta}_{-} \Gamma_{c d} \partial_{k} \theta_{-}\right) \\
& \times\left(11\left(\partial_{j} \bar{\theta}_{+} \Gamma_{11} \theta_{-}\right) \eta^{a c} \eta^{b d}+2\left(-\partial_{j} \bar{\theta}_{+} \Gamma^{c d} \Gamma^{a b} \Gamma_{11} \theta_{-}\right.\right. \\
& +\bar{\theta}_{-} \Gamma^{c} \Gamma^{a b} \Gamma^{d} \Gamma_{11} \partial_{j} \theta_{+} \\
& \left.\left.-\bar{\theta}_{+} \Gamma^{c} \Gamma^{a b} \Gamma^{d} \Gamma_{11} \partial_{j} \theta_{-}\right)\right)+3\left(\bar{\theta}_{-} \Gamma_{\mu c} \Gamma_{11} \partial_{j} \theta_{-}\right) \\
& \left.\times\left(\partial_{k} \bar{\theta}_{+} \Gamma^{\mu a b c} \theta_{-}+2\left(\partial_{k} \bar{\theta}_{+} \Gamma^{\mu b} \theta_{-}\right) \eta^{a c}\right)\right]
\end{aligned}
$$

\section{References}

1. N. Berkovits, Super-Poincaré covariant quantization of the superstring. JHEP 04, 018 (2000). arXiv:hep-th/0001035

2. N. Berkovits, Cohomology in the pure spinor formalism for the superstring. JHEP 09, 046 (2000). arXiv:hep-th/0006003

3. N. Berkovits, Covariant quantization of the superparticle using pure spinors. JHEP 09, 016 (2001). arXiv:hep-th/0105050

4. N. Berkovits, ICTP lectures on covariant quantization of the superstring. ICTP Lect. Notes Ser. 13, 57 (2003). arXiv:hep-th/0209059

5. O. Chandía, B.C. Vallilo, Conformal invariance of the pure spinor superstring in a curved background. JHEP 0404, 041 (2004). arXiv:hep-th/0401226

6. O. Chandía, A note on the classical BRST symmetry of the pure spinor string in a curved background. JHEP 0607, 019 (2006). arXiv:hep-th/0604115
7. O.A. Bedoya, O. Chandía, One loop conformal invariance of the type II pure spinor superstring in a curved background. JHEP 0701, 042 (2007). arXiv:hep-th/0609161

8. J. Kluson, Note about redefinition of BRST operator for pure spinor strings in general background. Phys. Rev. D 78, 066011 (2008). arXiv:0803.4390 [hep-th]

9. C. Mafra, Superstring scattering amplitudes with the pure spinor formalism. PhD Thesis, arXiv:0902.1552 [hep-th]

10. C. Mafra, C. Stahn, The one-loop open superstring massless fivepoint amplitude with the non-minimal pure spinor formalism. JHEP 0903, 126 (2009). arXiv:0902.1539 [hep-th]

11. N. Berkovits, C. Mafra, Equivalence of the two-loop superstring amplitudes in the pure spinor and RNS formalisms. Phys. Rev. Lett. 96, 011602 (2006). arXiv:hep-th/0509234

12. N. Berkovits, C. Mafra, Some superstring amplitude computations with the non-minimal pure spinor formalism. JHEP 0611, 079 (2006). arXiv:hep-th/0607187

13. C. Mafra, O. Schlotterer, Cohomology foundations of one-loop amplitudes in pure spinor superspace. arXiv:1408.3605 [hep-th]

14. J. Gomis, H. Ooguri, Nonrelativistic closed string theory. J. Math. Phys. 42, 3127 (2001). arXiv:hep-th/0009181

15. J. Brugues, T. Curtright, J. Gomis, L. Mezincescu, Non-relativistic strings and branes as non-linear realizations of Galilei groups. Phys. Lett. B 594, 227 (2004). arXiv:hep-th/0404175

16. J. Gomis, F. Passerini, T. Ramirez, A. van Proeyen, Non relativistic Dp branes. JHEP 0510, 007 (2005). arXiv:hep-th/0507135

17. K. Kamimura, T. Ramirez, Brane dualities in non-relativistic limit JHEP 0603, 058 (2006). arXiv:hep-th/0512146

18. M. Aganagic, C. Popescu, J.H. Schwarz, D-brane actions with local kappa symmetry. Phys. Lett. B 353, 311 (1997). arXiv:hep-th/9610249

19. M. Aganagic, C. Popescu, J.H. Schwarz, Gauge invariant and gauge fixed D-brane actions. Nucl. Phys. B 495, 99 (1997). arXiv:hep-th/9612080

20. M. Hatsuda, K. Kamimura, Wess-Zumino actions for IIA Dbranes and their supersymmetries. Nucl. Phys. B 535, 499 (1998). arXiv:hep-th/9804087

21. P.A. Grassi, G. Policastro, P. van Nieuwenhuisen, An introduction to the covariant quantization of the superstring. Class. Quant. Gravit. 20, S395 (2003). arXiv:hep-th/0302147

22. P.A. Grassi, G. Policastro, P. van Nieuwenhuisen, Yang-Mills theory as an illustration of the covariant quantization of superstrings. In 3rd International Sakharov Conference on Physics, 24-29 June (Russia, Moscow, 2002). arXiv:hep-th/0211095

23. J. Hoogeveen, Fundamentals of the Pure Spinor Formalism (Amsterdam University Press, Amsterdam, 2010)

24. L. Anguelova, P.A. Grassi, Super D-branes from BRST symmetry. JHEP 0311, 010 (2003). arXiv:hep-th/0307260

25. L. Brink, M. Henneaux, Principles of String Theory (Plenum Press, New York, 1988)

26. N. Berkovits, T. Fleury, Harmonic superspace from the $A d S_{5} \times$ $S^{5}$ pure spinor formalism. JHEP 03, 022 (2013). arXiv:1212.3296 [hep-th]

27. A. Mikhailov, A. Schwarz, R. Xu, Cohomology ring of the BRST operator associated to the sum of two pure spinors. Mod. Phys. Lett. A 28(23), 1350107 (2013). arXiv:1305.0071 [hep-th]

28. R. Xu, Cohomological Problems arising in Quantum Field Theory and String Theory. Ph.D. thesis, University of California, DAVIS

29. A. Mikhailov, R. Xu, BRST cohomology of the sum of two pure spinors. arXiv:1301.3353 [hep-th] 